\title{
Predictive formulation of the Nambu-Jona-Lasinio model
}

\author{
O. A. Battistel, ${ }^{1}$ G. Dallabona, ${ }^{2}$ and G. Krein ${ }^{3}$ \\ ${ }^{1}$ Departamento de Física, Universidade Federal de Santa Maria, 97119-900 Santa Maria, Rio Grande do Sul, Brazil \\ ${ }^{2}$ Departamento de Ciências Exatas, Universidade Federal de Lavras, Caixa Postal 37, 37200-000, Lavras, Minas Gerais, Brazil \\ ${ }^{3}$ Instituto de Física Teórica, Universidade Estadual Paulista, Rua Pamplona 145, 01405-900, São Paulo, Brazil
}

(Received 27 April 2007; revised manuscript received 18 January 2008; published 27 March 2008)

\begin{abstract}
A novel strategy to handle divergences typical of perturbative calculations is implemented for the Nambu-Jona-Lasinio model and its phenomenological consequences investigated. The central idea of the method is to avoid the critical step involved in the regularization process, namely, the explicit evaluation of divergent integrals. This goal is achieved by assuming a regularization distribution in an implicit way and making use, in intermediary steps, only of very general properties of such regularization. The finite parts are separated from the divergent ones and integrated free from effects of the regularization. The divergent parts are organized in terms of standard objects, which are independent of the (arbitrary) momenta running in internal lines of loop graphs. Through the analysis of symmetry relations, a set of properties for the divergent objects are identified, which we denominate consistency relations, reducing the number of divergent objects to only a few. The calculational strategy eliminates unphysical dependencies of the arbitrary choices for the routing of internal momenta, leading to ambiguity-free, and symmetry-preserving physical amplitudes. We show that the imposition of scale properties for the basic divergent objects leads to a critical condition for the constituent quark mass such that the remaining arbitrariness is removed. The model becomes predictive in the sense that its phenomenological consequences do not depend on possible choices made in intermediary steps. Numerical results are obtained for physical quantities at the one-loop level for the pion and sigma masses and pion-quark and sigmaquark coupling constants.
\end{abstract}

DOI: 10.1103/PhysRevD.77.065025

PACS numbers: 12.39. $-\mathrm{x}, 12.38 . \mathrm{Bx}, 11.30 . \mathrm{Rd}$

\section{INTRODUCTION}

It is largely accepted that many of the essential features of chiral symmetry in quantum chromodynamics (QCD) are captured by the Nambu-Jona-Lasinio (NJL) model [1], a simple relativistic quantum field theory with (nonrenormalizable) four-fermion interactions. In the limit of exact chiral symmetry the fermions are massless and the interaction Lagrangian density of the model, in its simplest version, contains the chirally symmetric sum of scalar and pseudoscalar four-fermion interactions. Since the first works, using the model with quark degrees of freedom, in the earlier days of QCD [2,3], the model has been extensively used to describe low energy hadronic observables, like hadronic masses, correlation and structure functions in vacuum and at finite densities and temperatures - for a complete list of references see the reviews in Refs. [4-11].

One of the reasons for the widespread use of the model is that it realizes the dynamical breaking of chiral symmetry already at the one-loop (mean field) approximation. The predictions of the model, however, are intimately compromising with the specific strategy adopted to handle the ultraviolet divergences given the nonrenormalizable nature of the model. As a consequence, the specification of a procedure for handling divergent amplitudes is a necessary and essential first step to be performed before extracting physical predictions as must be made in any relativistic quantum field theory. In a renormalizable theory, this is done by specifying a regularization procedure by which the divergences are isolated and eliminated through a conve- nient reparametrization of the theory, removing, in this way, any parameters introduced in the regularization process. Therefore, although essential and necessary, the regularization process plays a secondary role and seems to be a disposable intermediate step in the sense that it is not meant to modify the physical content of the theory. However, there is a distinctive and nontrivial aspect with the regularization of the ultraviolet divergences in the NJL model in view of its nonrenormalizability. Unlike with renormalizable models, as an increasing number of loops is considered, the reparametrization of the model can be made only at the cost of adding an increasing number of terms with extra coupling constants to the original Lagrangian in order to render physical amplitudes independent of the regularization procedure. In principle, there is no problem with a theory having an infinite number of coupling constants when using it as an effective field theory, as explained by Weinberg [12]. However, practitioners of the NJL model have followed the attitude of using it as a regularization-dependent model, considering the regularization procedure part of the definition of the model. Within such an attitude, a large body of interesting and valuable work has been and continues to be done using the model.

The regularization of divergent amplitudes is a delicate process due to the arbitrariness in the manipulation of improper integrals which can be converted into ambiguities when results become dependent on the choices involved. There are ambiguities associated with the arbitrary routing 
of the momenta in internal lines of divergent loop amplitudes, which invariably lead to the violation of space-time homogeneity. There are also ambiguities associated with the choice of the common scale for the divergent and finite parts of amplitudes that may lead to the breaking of scale invariance. In general, different sorts of ambiguities have the potential of leading to violations of symmetry relations of global and local gauge symmetries. The most commonly used regularization procedures for the NJL model such as the three- and four-momentum cutoff, Pauli-Villars, and proper-time lead to one or more of such symmetry violations. Dimensional regularization, although not much used within the NJL model, in general, leads to amplitudes free from ambiguities and symmetry preserving. However, it has problems at high densities and temperatures, when chiral symmetry is restored. This is due to the fact that the quadratic divergence which appears in almost all oneloop amplitudes must be assumed as zero in the zero-mass limit. Practice with the NJL model has shown that depending on the problem studied, one regularization scheme seems to be more appropriate than another because of the problems just mentioned. For example, when working with correlation functions, in general, dispersion relations are not automatically fulfilled in cutoff and proper-time regularizations, in contrast to Pauli-Villars regularization. On the other hand, while causality is preserved with PauliVillars regularization, unitarity is violated at high enough energies, although it is preserved with proper-time regularization. These sorts of problems are well known and arguments have been put forward and tricks invented to deal with such problems - for a discussion on these issues, see, for example, Refs. [13-15]. Obviously, this situation is unsatisfactory since one would like that the regularization scheme play a secondary role in the process of making predictions with the model.

The difficulties pointed out above lead Willey [16] and Gherghetta [17] to conclude that there is no way to make consistent physical predictions with the NJL model using traditional regularization techniques. However, this question in the context of the gauged NJL model was considered in a later work by Battistel and Nemes [18] using a novel strategy to handle divergent amplitudes [19]. The referred investigation revealed that NJL amplitudes, ambiguity-free and symmetry-preserving, can be obtained, and as such making the NJL model predictive. The central idea of the method is to avoid the critical step involved in the regularization process, namely, the explicit evaluation of divergent integrals. This goal is achieved by assuming a regularization distribution in an implicit way and making use, in intermediary steps, only of very general properties of such regularization. The finite parts are separated from the divergent ones and integrated free from effects of the regularization in a completely similar way as made in the treatment of renormalizable theories. The divergent parts are organized in terms of standard objects which are inde- pendent of the (arbitrary) momenta running in internal lines of loop graphs. Through the analysis of symmetry relations, a set of properties for the divergent objects is identified, which we denominate consistency relations (CR's), reducing the number of divergent objects to only a few ones. The remaining objects never really need to be evaluated. In renormalizable theories, they are eliminated by the counterterms. In a nonrenormalizable model, such as in the NJL, the basic divergences are fixed by fitting observables, as we will see along this contribution.

Having in mind this perspective, in the present work we extend the original discussion of Ref. [18] by presenting a complete and unified discussion on the symmetry relations involving Green's functions, including tensor operators. Some of the relations can be derived using the methods of current algebra, in particular, when using the conservation of the fermionic vector current and the proportionality of the divergence of the axial-vector current to the pseudoscalar current. Here we also discuss relations of Green's functions of tensor operators. Such relations cannot be obtained through current algebra methods, because the divergence of the fermionic tensor current cannot be written in terms of other fermionic currents. This is the case, for example, of the relations involving tensor-tensor twopoint amplitudes. In a first step of our investigation, we will show that it is possible to obtain physical amplitudes preserving the symmetries and automatically free from ambiguities associated with the arbitrariness in the routing of momenta in the internal lines of loops. In a second step, we will show that the undefined quantities associated with the divergent objects can be fixed phenomenologically, without the recourse of calculating any divergent integral, leading in this way to a regularization independent parametrization of the model. The model, within this formulation, becomes predictive in the sense that all arbitrariness is removed from the physical amplitudes. The model works like a "renormalizable" theory at the one-loop level.

The results obtained in the present paper are new and extend the applicability of the NJL model in a way that it becomes independent of a particular regularization scheme, since no explicit regularization is actually used. It is new because all symmetry constraints on general Green's functions, including tensor ones, are preserved and as such no problems with causality and unitarity can arise. The present method has also been applied in the context of CPT breaking in models with Chern-Simons interactions [20], to the neutral electromagnetic pion decay where the $A V V$ triangle anomaly phenomenon is discussed, triangle anomalies [21] and tensor densities [22]. In particular, it was shown that the adopted strategy furnishes the expected anomalous term and the ambiguities again play no relevant role [23]. One important aspect of the method presented here is that, since no explicit regulator is used, a result obtained within a given traditional regularization method can be immediately reobtained by 
explicit evaluation of the implicitly regulated expressions. With this, the use of the consistency relations allow one to identify the reasons why commonly used regularization schemes lead to symmetry violations. Invariably, the reasons are that not all the consistency relations are satisfied simultaneously within the traditional regularization methods.

The plan of this paper is the following. In Sec. II, we present the Lagrangian density of the NJL model used in this paper and discuss general "consistency constraints" that the one-loop Green's functions must satisfy in order not to violate symmetry relations. Next, in Sec. III we discuss a calculational scheme that preserves the general relationships among the Green's functions obtained in Sec. II. The calculational scheme isolates the purely divergent terms, which will disappear because of symmetry consideration or will be fitted to observables, while the finite parts are integrated without any regularization. In Sec. IV, we discuss the ambiguities associated with the choices for the momentum routing in the internal lines of loops and show that the methods used to isolate the divergent parts respect all the general relations among Green's functions. This aspect of the regularization is central to the paper and is highly nontrivial since, because of the ultraviolet divergences, these relations can very easily be violated when not being careful with the explicit evaluation of the integrals within a particular regularization scheme. In Sec. V, we identify the general properties that the divergent ambiguous quantities must satisfy in order to guarantee the preservation of fundamental symmetries. For that, Ward identities and other general constraints imposed by Furry's theorem will be invoked. The phenomenology and numerical results are presented in Sec. VI. Here, only the traditional observables, like pion and sigma masses and coupling constants are calculated to show that the traditional phenomenology is obtained in a straightforward way. Our conclusions and perspectives for future work are presented in Sec. VII.

\section{MODEL LAGRANGIAN AND CONSISTENCY CONSTRAINTS}

In this paper, we restrict the discussion to the simplest $\mathrm{SU}(2)$ version of the NJL model that incorporates the lightquark $u$ and $d$ flavors only. The SU(3) case will be considered elsewhere [24]. The Lagrangian density is given by

$$
\mathcal{L}=\bar{\psi}\left(i \not \partial-m_{0}\right) \psi+G_{S}\left[(\bar{\psi} \psi)^{2}+\left(\bar{\psi} \vec{\tau} i \gamma_{5} \psi\right)^{2}\right],
$$

where $\psi$ is the two-flavor, three-color quark field operator and $m_{0}$ is the diagonal current quark mass matrix. To simplify the discussion, we take equal $u$ and $d$ quark masses. The nonperturbative quark propagator $S(p)$ is given in terms of the self-energy $\Sigma(p)$ as

$$
S^{-1}(p)=\not p-\Sigma(p) \text {. }
$$

In the mean field approximation, the self-energy is mo- mentum independent $\Sigma(p) \equiv M$, with $M$ satisfying a gap equation [1]

$$
M=m_{0}-2 G_{S} N_{f}\langle\bar{\psi} \psi\rangle,
$$

where $N_{f}=2$ is the number of flavors, and $\langle\bar{\psi} \psi\rangle$ is the one-flavor, Lorentz scalar one-point function (the quark condensate) given by

$$
\begin{aligned}
\langle\bar{\psi} \psi\rangle & =-i \int \frac{d^{4} k}{(2 \pi)^{4}} \operatorname{Tr}[S(k)] \\
& =-4 N_{c} i \int \frac{d^{4} k}{(2 \pi)^{4}} \frac{M}{k^{2}-M^{2}},
\end{aligned}
$$

where $N_{c}=3$ is the number of colors.

In general, phenomenological predictions for meson masses and correlation functions require the evaluation of purely fermionic $n$-point Green's functions. In spite of the fact that the model is nonrenormalizable, the Green's functions obey well-defined relations among them. Such relations are the manifestation of the symmetries of the underlying Lagrangian defining the model. Therefore, in any attempt of describing a specific phenomenology it is crucial that the evaluation of physical amplitudes preserve such symmetry relations. If it turns out that such symmetry relations are not preserved by the calculation, the predictions cannot be characterized as consequences of the underlying symmetries supposed relevant for the specific phenomenology and which were the main motivation for using a schematic model like the NJL model.

A generic one-loop $n$-point Green's function can be defined as

$$
\begin{aligned}
T^{\Gamma_{1} \Gamma_{2} \cdots \Gamma_{n}}\left(k_{1}, k_{2}, \cdots, k_{n}\right)= & \int \frac{d^{4} k}{(2 \pi)^{4}} \operatorname{Tr}\left[\Gamma_{1} S\left(k+k_{1}\right)\right. \\
& \left.\times \Gamma_{2} S\left(k+k_{2}\right) \cdots \Gamma_{n} S\left(k+k_{n}\right)\right],
\end{aligned}
$$

where the trace is over Dirac indices only, and the $\Gamma_{i}$ represent one or more of the matrices $\left(1, \gamma_{\mu}, \gamma_{5}\right.$, $\left.\gamma_{\mu} \gamma_{5}, \sigma_{\mu \nu}\right)$ to which we attribute the labels $(S, V, P, A, T)$, respectively. The fermion propagators are given by Eq. (2) and the $k_{n}$ are arbitrary routing momenta in the internal lines, and are related to the external momenta. A physical amplitude can depend only on differences of $k_{n}$, any dependence on sums of $k_{n}$ is unphysical. We note that the highest superficial degree of divergence is cubic, and occurs for the amplitude with $n=1$. For $n>4$, the amplitudes are finite. In particular, in the NJL model the scalar one-point function is relevant for the gap equation, the two-point functions appear in the bound-state equations for mesons, the amplitudes with $n=3$ describe meson decays and the four-point functions are relevant for meson-meson scattering.

At the one-loop level, there appear only two divergences, a quadratic and the logarithmic. The cubic divergence is absent in the one-point functions either because of the 
trace or because the integral is identically zero-for the same reason there is no linear divergence in two- and threepoint functions. The standard procedure to deal with the divergences is to cut off the momentum integrals at some momentum $\Lambda$. With this, the model has two unknowns, the coupling $G_{S}$ and $\Lambda$. These can be fitted by using the values of the quark condensate $\langle\bar{\psi} \psi\rangle$, related to the quadratically divergent scalar one-point function $T^{S}$, and the pion-decay constant $f_{\pi}$, related to the logarithmically divergent axialvector pseudoscalar two-point function $T^{A P}$. Since these two types of divergences are the only ones that appear in all other Green's functions of the model, all divergences can be absorbed by the physical quantities $\langle\bar{\psi} \psi\rangle$ and $f_{\pi}$. In a certain sense, this is a type of renormalization.

There are two aspects we would like to note with respect to this "renormalization." First, one is explicitly using relations among divergent Green's functions having different numbers of points. Second, given the strict nonrenormalizability of the model, divergent amplitudes are related to physical quantities through a regularization function or, in the last instance, through the adjustment of regularization parameters which are interpreted as cutoffs in the momentum integration. It is well known that these two facts lead in general to symmetry violations. Moreover, the effects of the modification introduced in the regularization process remain present even in the finite parts of the amplitudes. In this respect, the regularization and renormalization procedure for nonrenormalizable and renormalizable models are treated in completely different manners. This discussion emphasizes the difficulties in satisfying kinematical constraints and symmetry relations involving one or more divergent amplitudes. The method we present here is based on the following strategy: (1) all constraints and symmetry relations are imposed without evaluating any divergent integral, (2) divergences are isolated into quantities that are independent of arbitrary routing momenta, and (3) amplitudes respecting all symmetry relations and free from ambiguities are obtained. All this is achieved without compromising with a particular regularization scheme.

Let us start considering the general "consistency constraints" that Green's functions must satisfy in order not to violate symmetry relations. One very general and powerful way to generate relations among Green's functions is to identify identities, at the integrand level, resulting from the contraction of the Lorentz vector indices of a vertex operator with an external momentum. Although this method is entirely equivalent to the current algebra technique for some types of amplitudes, it can be applied also to tensor currents - for which the methods of current algebra are not applicable. As an example, consider the identity

$$
\begin{gathered}
\left(k_{1}-k_{2}\right)^{\nu}\left[\gamma_{\mu} S\left(k+k_{1}\right) \gamma_{\nu} S\left(k+k_{2}\right)\right] \\
=\gamma_{\mu} S\left(k+k_{2}\right)-\gamma_{\mu} S\left(k+k_{1}\right),
\end{gathered}
$$

where $S(k)$ is the mean field quark operator given by
Eq. (2). This identity follows trivially from the algebra of the Dirac $\gamma$ matrices. Tracing both sides and integrating in momentum, a genuine relation among Green's functions of the model is obtained

$$
\left(k_{1}-k_{2}\right)^{\nu} T_{\mu \nu}^{V V}\left(k_{1}, k_{2}\right)=T_{\mu}^{V}\left(k_{1}\right)-T_{\mu}^{V}\left(k_{2}\right),
$$

where $T_{\mu}^{V}$ and $T_{\mu \nu}^{V V}$ are, respectively, the vector one-point function $\left(\Gamma_{1}=\gamma_{\mu}\right)$ and the two-point vector-vector amplitudes $\left(\Gamma_{1}=\gamma_{\mu}, \Gamma_{2}=\gamma_{\nu}\right)$, see Eq. (5). In the same way, one obtains

$$
\left(k_{1}-k_{2}\right)^{\mu} T_{\mu \nu}^{V V}\left(k_{1}, k_{2}\right)=T_{\nu}^{V}\left(k_{1}\right)-T_{\nu}^{V}\left(k_{2}\right) .
$$

Similarly, one can contract a Lorentz axial-vector twopoint density with an external momentum as

$$
\begin{aligned}
\left(k_{1}-\right. & \left.k_{2}\right)^{\mu}\left[\gamma_{\nu} \gamma_{5} S\left(k+k_{1}\right) \gamma_{\mu} \gamma_{5} S\left(k+k_{2}\right)\right] \\
= & 2 M\left[\gamma_{\nu} \gamma_{5} S\left(k+k_{1}\right) \gamma_{5} S\left(k+k_{2}\right)\right]+\gamma_{\nu} S\left(k+k_{2}\right) \\
& +\gamma_{\nu} \gamma_{5} S\left(k+k_{1}\right) \gamma_{5},
\end{aligned}
$$

where we have used the anticommutation of $\gamma_{5}$ and the $\gamma_{\nu}$ matrices. Again, taking the traces and integrating on both sides, we obtain

$$
\begin{aligned}
\left(k_{1}-k_{2}\right)^{\mu} T_{\nu \mu}^{A A}\left(k_{1}, k_{2}\right)= & 2 M T_{\nu}^{A P}\left(k_{1}, k_{2}\right)+T_{\nu}^{V}\left(k_{2}\right) \\
& -T_{\nu}^{V}\left(k_{1}\right),
\end{aligned}
$$

where the indices $A$ and $P$ stand for axial and pseudoscalar corresponding, respectively, to $F=\gamma_{\mu} \gamma_{5}$ and $\Gamma=\gamma_{5}$, as defined in the paragraph following Eq. (5). Following the procedure described above, we can get also the relations

$$
\begin{aligned}
& \left(k_{1}-k_{2}\right)^{\nu} T_{\nu}^{V S}\left(k_{1}, k_{2}\right)=T^{S}\left(k_{2}\right)-T^{S}\left(k_{1}\right), \\
& \left(k_{1}-k_{2}\right)^{\nu} T_{\nu}^{A P}\left(k_{1}, k_{2}\right)=-2 M T^{P P}\left(k_{1}, k_{2}\right)+T^{S}\left(k_{2}\right) \\
& -T^{S}\left(k_{1}\right) \text {, } \\
& \left(k_{1}-k_{2}\right)^{\mu} T_{\mu \nu}^{A V}\left(k_{1}, k_{2}\right)=-2 M T_{\nu}^{P V}\left(k_{1}, k_{2}\right)+T_{\nu}^{A}\left(k_{2}\right) \\
& -T_{\nu}^{A}\left(k_{1}\right), \\
& \left(k_{1}-k_{2}\right)^{\nu} T_{\mu \nu}^{A V}\left(k_{1}, k_{2}\right)=T_{\nu}^{A}\left(k_{2}\right)-T_{\nu}^{A}\left(k_{1}\right) .
\end{aligned}
$$

The expressions (11)-(14) are nothing more than the relations that follow from current algebra methods when using the conservation of the fermionic vector current and the proportionality of the divergence of the axial-vector current with the pseudoscalar current. The expressions involving the one-point functions correspond to the current commutator terms. Additional relations can be identified at the trace level:

$$
T_{\mu \nu}^{A V}\left(k_{1}, k_{2}\right)=\frac{i}{2 M} \varepsilon_{\mu \nu \lambda \sigma}\left(k_{1}-k_{2}\right)^{\lambda}\left(T^{S V}\right)^{\sigma}\left(k_{1}, k_{2}\right),
$$




$$
\begin{aligned}
T_{\mu}^{A P}\left(k_{1}, k_{2}\right)= & -\frac{1}{2 M}\left(k_{1}-k_{2}\right)_{\mu}\left[T^{S S}\left(k_{1}, k_{2}\right)\right. \\
& \left.+T^{P P}\left(k_{1}, k_{2}\right)\right] \\
T_{\mu \nu}^{V V}\left(k_{1}, k_{2}\right)- & T_{\mu \nu}^{A A}\left(k_{1}, k_{2}\right)= \\
& g_{\mu \nu}\left[T^{S S}\left(k_{1}, k_{2}\right)\right. \\
& \left.+T^{P P}\left(k_{1}, k_{2}\right)\right] .
\end{aligned}
$$

One of the advantages of the method used above is that it can be used to obtain relations for Green's functions involving tensor operators. Such relations cannot be obtained through current algebra methods simply because the divergence of the fermionic tensor current cannot be written in terms of other fermionic currents. This is the case of relations involving tensor-tensor two-point amplitudes, like, for example, the following one:

$$
\begin{aligned}
\left(k_{1}-\right. & \left.k_{2}\right)^{\mu} T_{\mu \nu \alpha \beta}^{T T}\left(k_{1}, k_{2}\right) \\
= & -g_{\alpha \nu}\left[T_{\beta}^{V}\left(k_{2}\right)-T_{\beta}^{V}\left(k_{1}\right)+\left(k_{1}-k_{2}\right)_{\beta} T^{S S}\left(k_{1}, k_{2}\right)\right] \\
& +g_{\beta \nu}\left[T_{\alpha}^{V}\left(k_{2}\right)-T_{\alpha}^{V}\left(k_{1}\right)+\left(k_{1}-k_{2}\right)_{\alpha} T^{S S}\left(k_{1}, k_{2}\right)\right] \\
& +\left(k_{1}-k_{2}\right)_{\alpha} T_{\nu \beta}^{A A}\left(k_{1}, k_{2}\right)-\left(k_{1}-k_{2}\right)_{\beta} T_{\nu \alpha}^{A A}\left(k_{1}, k_{2}\right) \\
& -2 M T_{\nu \alpha \beta}^{V T}\left(k_{1}, k_{2}\right) .
\end{aligned}
$$

Given the fact that three other Lorentz indexes are left uncontracted in the $T T$ two-point function, it is also possible to establish constraints on successive contractions with the external momenta. It is immediate to note that when these contractions involve both Lorentz indexes of a tensor operator, the result must vanish identically:

$$
\left(k_{1}-k_{2}\right)^{\alpha}\left(k_{1}-k_{2}\right)^{\beta} T_{\mu \nu \alpha \beta}^{T T}\left(k_{1}, k_{2}\right)=0 .
$$

This is due to the fact that $T_{\mu \nu \alpha \beta}^{T T} \sim \sigma_{\alpha \beta}$, where $\sigma_{\alpha \beta}=$ $-i / 2\left[\gamma_{\alpha}, \gamma_{\beta}\right]$, and therefore $\left(k_{1}-k_{2}\right)^{\alpha}\left(k_{1}-k_{2}\right)^{\beta} \sigma_{\alpha \beta}=$ 0 . This property imposes additional constraints on the consistent evaluation of the $T T$ two-point function. Even if such requirements seem to be obvious at this point, the divergent character of the integrals defining the amplitude makes satisfaction of this property far from being trivial.

Following strictly the same procedure outlined above, relations involving the remaining tensor two-point functions can be established as

$$
\begin{gathered}
\left(k_{1}-k_{2}\right)^{\alpha} T_{\mu \nu \alpha}^{T V}\left(k_{1}, k_{2}\right)=0, \\
\left(k_{1}-k_{2}\right)^{\mu} T_{\mu \nu}^{T P}\left(k_{1}, k_{2}\right)=-2 M T_{\nu}^{V P}\left(k_{1}, k_{2}\right), \\
\left(k_{1}-k_{2}\right)^{\alpha} T_{\mu \nu \alpha}^{T A}\left(k_{1}, k_{2}\right)=2 M T_{\mu \nu}^{T P}\left(k_{1}, k_{2}\right), \\
\left(k_{1}-k_{2}\right)^{\mu} T_{\mu \nu \alpha}^{T A}\left(k_{1}, k_{2}\right)= \\
=-2 M T_{\nu \alpha}^{V A}\left(k_{1}, k_{2}\right) \\
=-i \varepsilon_{\nu \alpha \lambda \xi}\left(k_{1}-k_{2}\right)^{\lambda} \\
\\
\times\left(T^{S V}\right)^{\xi}\left(k_{1}, k_{2}\right),
\end{gathered}
$$

$$
\begin{aligned}
\left(k_{1}-k_{2}\right)^{\mu} T_{\mu \nu}^{T S}\left(k_{1}, k_{2}\right)= & \frac{1}{2 m}\left\{\left(k_{1}-k_{2}\right)^{2} T_{\nu}^{V S}\left(k_{1}, k_{2}\right)\right. \\
& \left.-\left(k_{1}-k_{2}\right)_{\nu}\left[T^{S}\left(k_{2}\right)-T^{S}\left(k_{1}\right)\right]\right\} \\
= & -\frac{i}{2} \varepsilon_{\alpha \nu \lambda \xi}\left(k_{1}-k_{2}\right)^{\alpha}\left(T^{A V}\right)^{\lambda \dot{\xi}}\left(k_{1}, k_{2}\right),
\end{aligned}
$$

$$
\begin{aligned}
\left(k_{1}-k_{2}\right)^{\mu} T_{\mu \nu \alpha}^{T V}\left(k_{1}, k_{2}\right)= & \frac{1}{2 m}\left(k_{1}-k_{2}\right)^{2}\left[T_{\alpha \nu}^{V V}\left(k_{1}, k_{2}\right)\right. \\
& \left.-T_{\alpha \nu}^{A A}\left(k_{1}, k_{2}\right)\right] \\
& -\left(k_{1}-k_{2}\right)_{\nu} T_{\alpha}^{P A}\left(k_{1}, k_{2}\right) .
\end{aligned}
$$

In deriving these results, we have used the relations given in Eqs. (15)-(17).

At this point, it is very important to note that the results obtained above for the contraction of amplitudes with the external momentum are of general validity. Only algebraic manipulations have been made, no single divergent integral has been evaluated and no changes of variables under integration have been made. Thus, the results obtained are not compromised with any type of regularization. The important question one has to face when evaluating the integrals defining the different amplitudes is, how to give a meaning to the divergent integrals without violating these relations. This will be discussed in the next section.

\section{CALCULATIONAL SCHEME FOR HANDLING DIVERGENT INTEGRALS}

In the preceding section, we have considered the Green's functions for which one needs to construct a calculational scheme that preserves the general relationships among them, which we denominated consistency constraints. As a matter of consistency, all the constraints must be fulfilled without specifying special choices of the loop momenta. Divergences will appear in the form of improper Feynman integrals, but only a small number of them need to be evaluated since all the amplitudes are combinations of a few Feynman integrals.

The traditional way to handle a divergent Feynman integral is to adopt an explicit regularization. Invariably, this amounts to modifying the original integral in a way that the integration becomes well defined. In the context of NJL models, such modifications are commonly made by introducing in the integrand a distribution in the loop momentum to render the integral convergent. In doing so, the results of the integrals become a function of the parameters of the regulating distribution. In perturbatively renormalizable theories, one tries to isolate the purely divergent terms in order to specify the adequate counterterms for the renormalization. The parts which are independent of the regulating parameters are identified as the finite parts and carry the physical content of the amplitude. This means that the functions of the physical momenta are 
not affected by the regularization in the limit the regularization is removed. The procedure we adopt to use for the NJL model follows this general strategy closely, the purely divergent parts of the amplitudes will be fitted to observables, while the finite parts are integrated without any regularization.

In the first step, one assumes an unspecified regularization distribution $G_{\Lambda_{i}}\left(k^{2}, \Lambda_{i}^{2}\right)$ dependent on one or more "cutoff" parameters $\Lambda_{i}$, such that the original divergent integral is replaced by a finite one as [19]

$$
\begin{aligned}
\int \frac{d^{4} k}{(2 \pi)^{4}} f(k) & \rightarrow \int \frac{d^{4} k}{(2 \pi)^{4}} f(k) G_{\Lambda_{i}}\left(k^{2}, \Lambda_{i}^{2}\right) \\
& \equiv \int_{\Lambda} \frac{d^{4} k}{(2 \pi)^{4}} f(k) .
\end{aligned}
$$

The generic distribution $G\left(k^{2}, \Lambda_{i}^{2}\right)$, in addition to having the obvious property of turning the original integral convergent, must depend only on $k^{2}$ due to Lorentz invariance and must have the limit

$$
\lim _{\Lambda_{i}^{2} \rightarrow \infty} G_{\Lambda_{i}}\left(k^{2}, \Lambda_{i}^{2}\right)=1,
$$

which allows us to connect the regularized integral with the original one. Having assumed the existence of such a regularization distribution, one manipulates the integrand of the divergent integral in a way to isolate all the divergences in momentum-independent integrals. This goal can be achieved by using the identity

$$
\begin{aligned}
\frac{1}{\left[\left(k+k_{i}\right)^{2}-M^{2}\right]}= & \sum_{j=0}^{N} \frac{(-1)^{j}\left(k_{i}^{2}+2 k_{i} \cdot k\right)^{j}}{\left(k^{2}-M^{2}\right)^{j+1}} \\
& +\frac{(-1)^{N+1}\left(k_{i}^{2}+2 k_{i} \cdot k\right)^{N+1}}{\left(k^{2}-M^{2}\right)^{N+1}\left[\left(k+k_{i}\right)^{2}-M^{2}\right]},
\end{aligned}
$$

where the $k_{i}$ is (in principle) an arbitrary routing momentum of an internal line in a loop, and $M$ is the fermion mass running in the loop. The value of the integer $N$ is the smallest integer that makes integrals involving this last term finite when removing the regulating function. In view of Eq. (27), the corresponding integration can be performed without restrictions and will be free from the specific effects of an eventual regularization. No additional assumptions are made with respect to the remaining divergent terms.

When evaluating the amplitudes considered in the previous section, after taking the appropriate traces, it is not difficult to convince ourselves that only five divergent Feynman integrals will appear, namely

$$
\begin{aligned}
\left(I_{1} ; I_{1}^{\mu}\right) & =\int \frac{d^{4} k}{(2 \pi)^{4}} \frac{\left(1 ; k^{\mu}\right)}{\left[\left(k+k_{1}\right)^{2}-M^{2}\right]}, \\
\left(I_{2} ; I_{2}^{\mu} ; I_{2}^{\mu \nu}\right)= & \int \frac{d^{4} k}{(2 \pi)^{4}} \\
& \times \frac{\left(1 ; k^{\mu} ; k^{\mu} k^{\nu}\right)}{\left[\left(k+k_{1}\right)^{2}-M^{2}\right]\left[\left(k+k_{2}\right)^{2}-M^{2}\right]} .
\end{aligned}
$$

The divergent parts of these integrals can be rewritten in terms of five divergent quantities that we denote by $\square_{\alpha \beta \mu \nu}$, $\Delta_{\mu \nu}, \nabla_{\mu \nu}, I_{\mathrm{log}}$, and $I_{\text {quad }}$. Specifically, the integrals $I_{1}$ and $I_{1 \mu}$ that appear in the one-point amplitudes can be written as

$$
\begin{gathered}
I_{1}\left(k_{1}\right)=\left[I_{\text {quad }}\left(M^{2}\right)\right]+k_{1}^{\alpha} k_{1}^{\beta}\left[\Delta_{\alpha \beta}\right], \\
I_{1 \mu}\left(k_{1}\right)=-k_{1 \mu}\left[I_{\text {quad }}\left(M^{2}\right)\right]-k_{1}^{\beta}\left[\nabla_{\beta \mu}\right] \\
\\
-\frac{1}{3} k_{1}^{\beta} k_{1}^{\alpha} k_{1}^{\nu}\left[\square_{\alpha \beta \mu \nu}\right]-\frac{1}{3} k_{1 \mu} k_{1}^{\alpha} k_{1}^{\beta}\left[\Delta_{\alpha \beta}\right] \\
+\frac{1}{3} k_{1}^{2} k_{1}^{\alpha}\left[\Delta_{\alpha \mu}\right] .
\end{gathered}
$$

The integrals $I_{2}, I_{2 \mu}$, and $I_{2 \mu \nu}$ related to two-point amplitudes can be written as

$$
I_{2}\left(k_{1}, k_{2}\right)=\left[I_{\log }\left(M^{2}\right)\right]-i(4 \pi)^{-2}\left[Z_{0}\left(\left(k_{1}-k_{2}\right)^{2} ; M^{2}\right)\right],
$$

$$
I_{2 \mu}\left(k_{1}, k_{2}\right)=-\frac{1}{2}\left(k_{1}+k_{2}\right)^{\alpha}\left[\Delta_{\alpha \mu}\right]-\frac{1}{2}\left(k_{1}+k_{2}\right)_{\mu}\left(I_{2}\right)
$$

$$
\begin{aligned}
I_{2 \mu \nu}\left(k_{1}, k_{2}\right)= & \frac{1}{2}\left[\nabla_{\mu \nu}\right]-\frac{1}{12}\left(k_{1}-k_{2}\right)^{2}\left[\Delta_{\mu \nu}\right]+\frac{1}{6}\left(k_{2}^{\alpha} k_{2}^{\beta}+k_{1}^{\alpha} k_{2}^{\beta}+k_{1}^{\alpha} k_{1}^{\beta}\right)\left[\square_{\alpha \beta \mu \nu}\right]+\frac{1}{6}\left(k_{2 \nu} k_{2}^{\beta}+k_{1 \nu} k_{2}^{\beta}+k_{1 \nu} k_{1}^{\beta}\right)\left[\Delta_{\beta \mu}\right] \\
& +\frac{1}{6}\left(k_{2 \mu} k_{2}^{\beta}+k_{1 \mu} k_{2}^{\beta}+k_{1 \mu} k_{1}^{\beta}\right)\left[\Delta_{\beta \nu}\right]+\frac{1}{2} g_{\mu \nu}\left[I_{\text {quad }}\left(M^{2}\right)\right]-\frac{1}{12} g_{\mu \nu}\left(k_{1}-k_{2}\right)^{2}\left[I_{\log }\left(M^{2}\right)\right]+\frac{1}{6}\left(2 k_{2 \nu} k_{2 \mu}+k_{1 \nu} k_{2 \mu}\right. \\
& \left.+k_{1 \mu} k_{2 \nu}+2 k_{1 \nu} k_{1 \mu}\right)\left[I_{\log }\left(M^{2}\right)\right]+i(4 \pi)^{-2}\left[\left(k_{1}-k_{2}\right)_{\mu}\left(k_{1}-k_{2}\right)_{\nu}-g_{\mu \nu}\left(k_{1}-k_{2}\right)^{2}\right]\left[\frac{1}{4} Z_{0}\left(\left(k_{1}-k_{2}\right)^{2} ; M^{2}\right)\right. \\
& \left.-Z_{2}\left(\left(k_{1}-k_{2}\right)^{2} ; M^{2}\right)\right]-i(4 \pi)^{-2}\left(k_{1}+k_{2}\right)_{\mu}\left(k_{1}+k_{2}\right)_{\nu}\left[\frac{1}{4} Z_{0}\left(\left(k_{1}-k_{2}\right)^{2} ; M^{2}\right)\right] .
\end{aligned}
$$

In these, the functions $Z_{0}\left(q^{2} ; M^{2}\right)$ and $Z_{2}\left(q^{2} ; M^{2}\right)$ are finite and can be written generically as

$$
Z_{k}\left(q^{2} ; M^{2}\right)=\int_{0}^{1} d z z^{k} \log \left[\frac{q^{2} z(1-z)-M^{2}}{-M^{2}}\right]
$$

and $\square_{\alpha \beta \mu \nu}, \Delta_{\mu \nu}, \nabla_{\mu \nu}, I_{\log }$ and $I_{\text {quad }}$ are momentum-independent divergent quantities given by 


$$
\begin{aligned}
& \square_{\alpha \beta \mu \nu}= \int_{\Lambda} \frac{d^{4} k}{(2 \pi)^{4}} \frac{24 k_{\mu} k_{\nu} k_{\alpha} k_{\beta}}{\left(k^{2}-M^{2}\right)^{4}}-g_{\alpha \beta} \int_{\Lambda} \frac{d^{4} k}{(2 \pi)^{4}} \\
& \times \frac{4 k_{\mu} k_{\nu}}{\left(k^{2}-M^{2}\right)^{3}}-g_{\alpha \nu} \int_{\Lambda} \frac{d^{4} k}{(2 \pi)^{4}} \frac{4 k_{\beta} k_{\mu}}{\left(k^{2}-M^{2}\right)^{3}} \\
&-g_{\alpha \mu} \int_{\Lambda} \frac{d^{4} k}{(2 \pi)^{4}} \frac{4 k_{\beta} k_{\nu}}{\left(k^{2}-M^{2}\right)^{3}}, \\
& \Delta_{\mu \nu}= \int_{\Lambda} \frac{d^{4} k}{(2 \pi)^{4}} \frac{4 k_{\mu} k_{\nu}}{\left(k^{2}-M^{2}\right)^{3}}-\int_{\Lambda} \frac{d^{4} k}{(2 \pi)^{4}} \frac{g_{\mu \nu}}{\left(k^{2}-M^{2}\right)^{2}} \\
& \nabla_{\mu \nu}= \int_{\Lambda} \frac{d^{4} k}{(2 \pi)^{4}} \frac{2 k_{\nu} k_{\mu}}{\left(k^{2}-M^{2}\right)^{2}}-\int_{\Lambda} \frac{d^{4} k}{(2 \pi)^{4}} \frac{g_{\mu \nu}}{\left(k^{2}-M^{2}\right)} \\
& I_{\mathrm{quad}}\left(M^{2}\right)=\int_{\Lambda} \frac{d^{4} k}{(2 \pi)^{4}} \frac{1}{\left(k^{2}-M^{2}\right)} \\
& I_{\log }\left(M^{2}\right)=\int_{\Lambda} \frac{d^{4} k}{(2 \pi)^{4}} \frac{1}{\left(k^{2}-M^{2}\right)^{2}},
\end{aligned}
$$

Since the one-point functions are purely divergent, they can be expressed entirely in terms of (a subset of) the above divergent quantities as

$$
\begin{gathered}
T^{S}\left(k_{1}\right)=4 M\left\{\left[I_{\text {quad }}\left(M^{2}\right)\right]+k_{1}^{\alpha} k_{1}^{\beta}\left[\Delta_{\beta \alpha}\right]\right\}, \\
T_{\mu}^{V}\left(k_{1}\right)=4\left\{-k_{1}^{\beta}\left[\nabla_{\beta \mu}\right]-\frac{1}{3} k_{1}^{\beta} k_{1}^{\alpha} k_{1}^{\nu}\left[\square_{\alpha \beta \mu \nu}\right]+\frac{1}{3} k_{1}^{2} k_{1}^{\nu}\left[\Delta_{\mu \nu}\right]\right. \\
\left.+\frac{2}{3} k_{1 \mu} k_{1}^{\alpha} k_{1}^{\beta}\left[\Delta_{\alpha \beta}\right]\right\} .
\end{gathered}
$$

The two-point functions contain finite and divergent parts, and can be written as

$$
\begin{aligned}
T^{S S}\left(k_{1}, k_{2}\right)= & 4\left\{\left[I_{\text {quad }}\left(M^{2}\right)\right]+\frac{1}{2}\left[4 M^{2}-\left(k_{1}-k_{2}\right)^{2}\right.\right. \\
& \times\left[I_{\log }\left(M^{2}\right)\right]-i(4 \pi)^{-2}\left[4 M^{2}-\left(k_{1}-k_{2}\right)^{2}\right] \\
& \left.\times\left[\frac{1}{2} Z_{0}\left(\left(k_{1}-k_{2}\right)^{2}, M^{2}\right)\right]\right\}+\left(k_{1}-k_{2}\right)^{\alpha} \\
& \times\left(k_{1}-k_{2}\right)^{\beta}\left[\Delta_{\alpha \beta}\right]+\left(k_{1}+k_{2}\right)^{\alpha} \\
& \times\left(k_{1}+k_{2}\right)^{\beta}\left[\Delta_{\alpha \beta}\right],
\end{aligned}
$$

$$
\begin{aligned}
T^{P P}\left(k_{1}, k_{2}\right)= & 4\left\{-\left[I_{\text {quad }}\left(M^{2}\right)+\frac{1}{2}\left(k_{1}-k_{2}\right)^{2}\left[I_{\log }\left(M^{2}\right)\right]\right.\right. \\
& \left.-i(4 \pi)^{-2}\left(k_{1}-k_{2}\right)^{2}\left[\frac{1}{2} Z_{0}\left(\left(k_{1}-k_{2}\right)^{2}, M^{2}\right)\right]\right\} \\
& -\left(k_{1}-k_{2}\right)^{\alpha}\left(k_{1}-k_{2}\right)^{\beta}\left[\Delta_{\alpha \beta}\right] \\
& -\left(k_{1}+k_{2}\right)^{\alpha}\left(k_{1}+k_{2}\right)^{\beta}\left[\Delta_{\alpha \beta}\right],
\end{aligned}
$$

$$
\begin{aligned}
& T_{\mu}^{P A}\left(k_{1}, k_{2}\right)= 4 M\left(k_{1}-k_{2}\right)_{\mu}\left\{\left[I_{\log }\left(M^{2}\right)\right]\right. \\
&\left.-i(4 \pi)^{-2}\left[Z_{0}\left(\left(k_{1}-k_{2}\right)^{2}, M^{2}\right)\right]\right\}, \\
& T_{\mu}^{V S}\left(k_{1}, k_{2}\right)=-4 M\left(k_{1}+k_{2}\right)^{\xi}\left[\Delta_{\xi \mu}\right],
\end{aligned}
$$$$
T_{\mu \nu}^{A V}\left(k_{1}, k_{2}\right)=-2 i \varepsilon_{\mu \nu \alpha \beta}\left(k_{2}-k_{1}\right)^{\beta}\left(k_{1}+k_{2}\right)^{\xi}\left[\Delta_{\xi}^{\alpha}\right],
$$

$$
\begin{aligned}
T_{\mu \nu}^{V V}\left(k_{1}, k_{2}\right)= & \frac{4}{3}\left[\left(k_{1}-k_{2}\right)^{2} g_{\mu \nu}-\left(k_{1}-k_{2}\right)_{\mu}\left(k_{1}-k_{2}\right)_{\nu}\right] \\
& \times\left\{\left[I_{\log }\left(M^{2}\right)\right]-i(4 \pi)^{-2}\right. \\
& \times\left[\frac{1}{3}+\frac{\left(2 M^{2}+\left(k_{1}-k_{2}\right)^{2}\right)}{\left(k_{1}-k_{2}\right)^{2}}\right. \\
& \left.\left.\times\left[Z_{0}\left(\left(k_{1}-k_{2}\right)^{2}, M^{2}\right)\right]\right]\right\}+A_{\mu \nu}
\end{aligned}
$$

$$
\begin{aligned}
T_{\mu \nu}^{A A}\left(k_{1}, k_{2}\right)= & \frac{4}{3}\left[\left(k_{1}-k_{2}\right)^{2} g_{\mu \nu}-\left(k_{1}-k_{2}\right)_{\mu}\left(k_{1}-k_{2}\right)_{\nu}\right] \\
& \times\left\{\left[I_{\log }\left(M^{2}\right)\right]-i(4 \pi)^{-2}\right. \\
& \times\left[\frac{1}{3}+\frac{\left(2 M^{2}+\left(k_{1}-k_{2}\right)^{2}\right)}{\left(k_{1}-k_{2}\right)^{2}}\right. \\
& \left.\left.\times\left[Z_{0}\left(\left(k_{1}-k_{2}\right)^{2}, M^{2}\right)\right]\right]\right\}-8 M^{2} g_{\mu \nu}\left\{\left[I_{\log }\left(M^{2}\right)\right]\right. \\
& \left.-i(4 \pi)^{-2}\left[Z_{0}\left(\left(k_{1}-k_{2}\right)^{2}, M^{2}\right)\right]\right\}+A_{\mu \nu}, \quad(50)
\end{aligned}
$$

$$
\begin{aligned}
T_{\mu \nu}^{S T}\left(k_{1}, k_{2}\right)= & -2\left\{\left(k_{1}-k_{2}\right)_{\mu}\left(k_{1}+k_{2}\right)^{\xi}\left[\Delta_{\xi \nu}\right]\right. \\
& \left.-\left(k_{1}-k_{2}\right)_{\nu}\left(k_{1}+k_{2}\right)^{\xi}\left[\Delta_{\xi \mu}\right]\right\}
\end{aligned}
$$

$$
\begin{gathered}
T_{\mu \nu}^{P T}\left(k_{1}, k_{2}\right)=-2 i \varepsilon_{\mu \nu \alpha \beta}\left(k_{1}-k_{2}\right)^{\alpha}\left(k_{1}+k_{2}\right)^{\xi}\left[\Delta_{\xi}^{\beta}\right], \\
T_{\alpha \mu \nu}^{A T}\left(k_{1}, k_{2}\right)=4 i \varepsilon_{\mu \nu \alpha \beta}\left(k_{1}+k_{2}\right)^{\xi}\left[\Delta_{\xi}^{\beta}\right],
\end{gathered}
$$

$$
\begin{aligned}
T_{\alpha \mu \nu}^{V T}\left(k_{1}, k_{2}\right)= & 4 M\left(g_{\alpha \nu} g_{\mu \lambda}-g_{\alpha \mu} g_{\nu \lambda}\right)\left(k_{1}-k_{2}\right)^{\lambda} \\
& \times\left\{\left[I_{\log }\left(M^{2}\right)\right]-i(4 \pi)^{-2}\right. \\
& \left.\times\left[Z_{0}\left(\left(k_{1}-k_{2}\right)^{2}, M^{2}\right)\right]\right\}
\end{aligned}
$$




$$
\begin{aligned}
T_{\alpha \beta \mu \nu}^{T T}\left(k_{1}, k_{2}\right)= & \left(g_{\alpha \mu} g_{\beta \xi} g_{\nu \lambda}-g_{\alpha \nu} g_{\beta \xi} g_{\mu \lambda}+g_{\beta \nu} g_{\alpha \xi} g_{\mu \lambda}-g_{\beta \mu} g_{\alpha \xi} g_{\nu \lambda}\right) \frac{4}{3}\left[\left(k_{1}-k_{2}\right)^{2} g^{\xi \lambda}-\left(k_{1}-k_{2}\right)^{\xi}\left(k_{1}-k_{2}\right)^{\lambda}\right] \\
& \times\left\{I_{\log }\left(m^{2}\right)-i(4 \pi)^{-2}\left[\frac{1}{3}+\frac{2 M^{2}+\left(k_{1}-k_{2}\right)^{2}}{\left(k_{1}-k_{2}\right)^{2}}\left[Z_{0}\left(M^{2},\left(k_{1}-k_{2}\right)^{2}\right)\right]\right]\right\}+4\left(g_{\alpha \nu} g_{\beta \mu}-g_{\alpha \mu} g_{\beta \nu}\right) \\
& \times\left\{-\left[I_{\text {quad }}\left(M^{2}\right)\right]+\frac{1}{2}\left[4 M^{2}+\left(k_{1}-k_{2}\right)^{2}\right]\left[I_{\log }\left(M^{2}\right)\right]-\frac{1}{2}\left[4 M^{2}+\left(k_{1}-k_{2}\right)^{2}\right] i(4 \pi)^{-2}\left[Z_{0}\left(\left(k_{1}-k_{2}\right)^{2}, M^{2}\right)\right]\right. \\
& \left.-\frac{1}{4}\left(k_{1}-k_{2}\right)^{\lambda}\left(k_{1}-k_{2}\right)^{\xi}\left[\Delta_{\lambda \xi}\right]-\frac{1}{4}\left(k_{1}+k_{2}\right)^{\lambda}\left(k_{1}+k_{2}\right)^{\xi}\left[\Delta_{\lambda \xi}\right]\right\} \\
& +g_{\alpha \mu} A_{\beta \nu}-g_{\alpha \nu} A_{\beta \mu}+g_{\beta \nu} A_{\alpha \mu}-g_{\beta \mu} A_{\alpha \nu} .
\end{aligned}
$$

In the above, we have defined $A_{\mu \nu}$ as

$$
\begin{aligned}
A_{\mu \nu}= & 4\left[\nabla_{\mu \nu}\right]+\left(k_{1}-k_{2}\right)^{\alpha}\left(k_{1}-k_{2}\right)^{\beta}\left[\frac{1}{3} \square_{\alpha \beta \mu \nu}\right. \\
& \left.+\frac{1}{3} g_{\alpha \nu} \Delta_{\mu \beta}+g_{\alpha \mu} \Delta_{\beta \nu}-g_{\mu \nu} \Delta_{\alpha \beta}-\frac{2}{3} g_{\alpha \beta} \Delta_{\mu \nu}\right] \\
& +\left[\left(k_{1}-k_{2}\right)^{\alpha}\left(k_{1}+k_{2}\right)^{\beta}-\left(k_{1}+k_{2}\right)^{\alpha}\left(k_{1}-k_{2}\right)^{\beta}\right] \\
& \times\left[\frac{1}{3} \square_{\alpha \beta \mu \nu}+\frac{1}{3} g_{\nu \alpha} \Delta_{\mu \beta}+\frac{1}{3} g_{\alpha \mu} \Delta_{\beta \nu}\right] \\
& +\left(k_{1}+k_{2}\right)^{\alpha}\left(k_{1}+k_{2}\right)^{\beta}\left[\square_{\alpha \beta \mu \nu}-g_{\mu \beta} \Delta_{\nu \alpha}\right. \\
& \left.-g_{\alpha \mu} \Delta_{\beta \nu}-3 g_{\mu \nu} \Delta_{\alpha \beta}\right] .
\end{aligned}
$$

The remaining one- and two-point functions not considered above are identically zero due to vanishing Dirac traces.

At this point, it is important to note the generality of the method. No momentum shifts were done and no single divergent integral was calculated. For this reason, the results obtained can be used with any preferred regularization method. Of course, different regularization schemes can lead to ambiguities and violation of symmetry relations between Green's functions, as we shall discuss in the next section.

\section{AMBIGUITIES AND THE CONSISTENCY CONSTRAINTS}

In the preceding section, we have completed the evaluation of all fermionic one- and two-point amplitudes. Before any further steps in evaluating these amplitudes, it is important to notice that there are ambiguities associated with the choices for the momentum routing in the internal lines of loops. In the calculations performed in the previous section, we have not made any momentum shifts in intermediary steps and have left the labels of the internal momenta $k_{1}$ and $k_{2}$ completely arbitrary and unspecified. However, due to momentum conservation, a physical amplitude can depend only on the difference $q \equiv k_{1}-k_{2}$, and no dependence on the sum $Q \equiv k_{1}+k_{2}$ can be present in the amplitude. There can be no dependence on $Q$ because this combination is ambiguous: two different choices of $k_{1}$ and $k_{2}$ can give the same $q$, but they will always give different $Q$ 's. In view of this, one could imagine that it is impossible to make any predictions because the amplitudes contain dependencies on arbitrary contributions which are functions of $Q$. In general, depending on the regularization scheme used, this is actually true. In the literature associated with the NJL model, it is usual to use a particular routing, the symmetric combination $k_{1}=q / 2$ and $k_{2}=$ $-q / 2$, meaning that $Q=0$ and the amplitudes are automatically free of ambiguities. However, restriction to one particular choice, besides breaking homogeneity of spacetime, also leads to difficulties with amplitudes containing more than two propagators where there are in principle more than two arbitrary momenta and choices, like the above would not be allowed because in general it would lead to violations of momentum conservation.

Our aim now is to show that what we have done so far does not lead to violations of the relations among Green's functions because of arbitrary choices of the momenta $k_{1}$ and $k_{2}$. As shown in Sec. II, such relations are very general, but due to the ultraviolet divergences they can very easily be violated when not being careful with the explicit evaluation of the integrals within a particular regularization scheme.

It is a simple task to identify the ambiguous terms, proportional to $Q$. In the one-point functions they are given by (note that for these $Q=k_{1}$ )

$$
\left[T^{S}\left(k_{1}\right)\right]_{\mathrm{ambi}}=4 M k_{1}^{\beta} k_{1}^{\alpha}\left[\Delta_{\beta \alpha}\right],
$$

$$
\begin{aligned}
{\left[T_{\mu}^{V}\left(k_{1}\right)\right]_{\mathrm{ambi}}=} & 4\left\{-k_{1}^{\beta}\left[\nabla_{\beta \mu}\right]-\frac{1}{3} k_{1}^{\beta} k_{1}^{\alpha} k_{1}^{\nu}\left[\square_{\alpha \beta \mu \nu}\right]\right. \\
& \left.+\frac{1}{3} k_{1}^{2} k_{1}^{\nu}\left[\Delta_{\mu \nu}\right]+\frac{2}{3} k_{1 \mu} k_{1}^{\alpha} k_{1}^{\beta}\left[\Delta_{\alpha \beta}\right]\right\} .
\end{aligned}
$$

In the two-point functions, the ambiguous terms are given by

$$
\begin{gathered}
{\left[T^{S S}\left(k_{1}, k_{2}\right)\right]_{\mathrm{ambi}}=Q^{\alpha} Q^{\beta}\left[\Delta_{\alpha \beta}\right],} \\
{\left[T^{P P}\left(k_{1}, k_{2}\right)\right]_{\mathrm{ambi}}=-\left[T^{S S}\left(k_{1}, k_{2}\right)\right]_{\mathrm{ambi}},} \\
{\left[T_{\mu}^{P A}\left(k_{1}, k_{2}\right)\right]_{\mathrm{ambi}}=0,} \\
{\left[T_{\mu}^{V S}\left(k_{1}, k_{2}\right)\right]_{\mathrm{ambi}}=-4 M Q^{\xi}\left[\Delta_{\xi \mu}\right],} \\
{\left[T_{\mu \nu}^{A V}\left(k_{1}, k_{2}\right)\right]_{\mathrm{ambi}}=2 i \varepsilon_{\mu \nu \alpha \beta} q^{\beta} Q^{\xi}\left[\Delta_{\xi}^{\alpha}\right],}
\end{gathered}
$$




$$
\begin{gathered}
{\left[T_{\mu \nu}^{V V}\left(k_{1}, k_{2}\right)\right]_{\mathrm{ambi}}=\left[q^{\alpha} Q^{\beta}-Q^{\alpha} q^{\beta}\right]\left[\frac{1}{3} \square_{\alpha \beta \mu \nu}+\frac{1}{3} g_{\nu \alpha} \Delta_{\mu \beta}\right.} \\
\left.+\frac{1}{3} g_{\alpha \mu} \Delta_{\beta \nu}\right]+Q^{\alpha} Q^{\beta}\left[\square_{\alpha \beta \mu \nu}\right. \\
\left.-g_{\mu \beta} \Delta_{\nu \alpha}-g_{\alpha \mu} \Delta_{\beta \nu}-3 g_{\mu \nu} \Delta_{\alpha \beta}\right], \\
{\left[T_{\mu \nu}^{A A}\left(k_{1}, k_{2}\right)\right]_{\mathrm{ambi}}=\left[T_{\mu \nu}^{V V}\left(k_{1}, k_{2}\right)\right]_{\mathrm{ambi}},} \\
{\left[T_{\mu \nu}^{S T}\left(k_{1}, k_{2}\right)\right]_{\mathrm{ambi}}=-2\left\{q_{\mu} Q^{\xi}\left[\Delta_{\xi \nu}\right]-q_{\nu} Q^{\xi}\left[\Delta_{\xi \mu}\right]\right\},} \\
{\left[T_{\mu \nu}^{P T}\left(k_{1}, k_{2}\right)\right]_{\mathrm{ambi}}=-i 2 \varepsilon_{\mu \nu \alpha \beta} q^{\alpha} Q^{\xi}\left[\Delta_{\xi}^{\beta}\right],} \\
{\left[T_{\alpha \mu \nu}^{V T}\left(k_{1}, k_{2}\right)\right]_{\mathrm{ambi}}=4 i M \varepsilon_{\mu \nu \alpha \beta} Q^{\xi}\left[\Delta_{\xi}^{\beta}\right],} \\
{\left[T_{\alpha \mu \nu}^{V T}\left(k_{1}, k_{2}\right)\right]_{\mathrm{ambi}}=0,} \\
{\left[T_{\alpha \beta \mu \nu}^{T T}\left(k_{1}, k_{2}\right)\right]_{\mathrm{ambi}}=-\frac{1}{4} Q^{\lambda} Q^{\xi}\left[\Delta_{\lambda \xi}\right]+g_{\alpha \mu}\left(T_{\beta \nu}^{V V}\right)_{\mathrm{ambi}}} \\
-g_{\alpha \nu}\left(T_{\beta \mu}^{V V}\right)_{\mathrm{ambi}}+g_{\beta \nu}\left(T_{\alpha \mu}^{V V}\right)_{\mathrm{ambi}} \\
-g_{\beta \mu}\left(T_{\alpha \nu}^{V V}\right)_{\mathrm{ambi}} .
\end{gathered}
$$

Note that all ambiguous terms appear as coefficients of the divergent quantities $\nabla_{\beta \mu}, \square_{\alpha \beta \mu \nu}$, and $\Delta_{\alpha \beta}$. These quantities also play an important role in the analysis of the algebraic relations involving different Green's functions, the consistency constraints obtained in Sec. II. Those constraints were obtained by making the only assumption of the validity of the linearity of the integration operation. Although this assumption seems very reasonable, the mathematical indefiniteness due to the divergences turns the preservation of such relations a nontrivial supposition.

Let us now show that, despite the amplitudes themselves containing ambiguous terms, the general relations involving different Green's functions obtained in Sec. II are not violated by the manipulations done so far. We start with the relations in Eqs. (7) and (8) involving the Green's functions $T_{\mu \nu}^{V V}\left(k_{1}, k_{2}\right)$ and $T_{\nu}^{V}\left(k_{1}\right)$. Contracting $T_{\mu \nu}^{V V}\left(k_{1}, k_{2}\right)$ given in Eq. (49) with the external momentum $q$ leads to

$$
q^{\mu} T_{\mu \nu}^{V V}\left(k_{1}, k_{2}\right)=q^{\mu} A_{\mu \nu} .
$$

From Eq. (8), one sees that one must identify on the righthand side (r.h.s.). of Eq. (71) the difference between two vector one-point functions corresponding to the internal propagator carrying arbitrary momenta $k_{1}$ and $k_{2}$. For this purpose, we take Eq. (56) for $A_{\mu \nu}$ and contract it with $q^{\mu}$

$$
q^{\mu} A_{\mu \nu}=T_{\nu}^{V}\left(k_{2}\right)-T_{\nu}^{V}\left(k_{1}\right),
$$

and also with $q^{\nu}$

$$
q^{\nu} A_{\mu \nu}=T_{\mu}^{V}\left(k_{2}\right)-T_{\mu}^{V}\left(k_{1}\right),
$$

where we used Eq. (43) for identifying $T_{\nu}^{V}\left(k_{1}\right)$. These results imply that the algebraic relations of Eqs. (7) and (8) involving $T_{\nu}^{V}\left(k_{1}\right)$ and $T_{\mu \nu}^{V V}\left(k_{1}, k_{2}\right)$ obtained by the for- mal manipulations in Sec. II are preserved by the explicit and independent evaluations of $T_{\mu \nu}^{V V}\left(k_{1}, k_{2}\right)$ and $T_{\mu}^{V}\left(k_{1}\right)$ in Sec. III. Therefore, the relations are preserved in spite of the fact that both amplitudes $T_{\nu}^{V}\left(k_{1}\right)$ and $T_{\mu \nu}^{V V}\left(k_{1}, k_{2}\right)$ have ambiguous pieces, which are proportional to the divergent quantities $\nabla_{\beta \mu}, \square_{\alpha \beta \mu \nu}$, and $\Delta_{\alpha \beta}$.

Next we consider the relation given in Eq. (11) involving the amplitudes $T_{\mu}^{V S}$ and $T^{S}$. Using the explicit expression for $T_{\mu}^{V S}\left(k_{1}, k_{2}\right)$ given in Eq. (47), one obtains

$$
\begin{aligned}
q^{\mu} T_{\mu}^{V S}\left(k_{1}, k_{2}\right)= & -4 M\left(k_{1}^{\mu} k_{1}^{\xi}+k_{1}^{\mu} k_{2}^{\xi}-k_{2}^{\mu} k_{1}^{\xi}-k_{2}^{\mu} k_{2}^{\xi}\right) \\
& \times\left[\Delta_{\xi \mu}\right] .
\end{aligned}
$$

Because of the obvious symmetry under interchange of the Lorentz indexes $\xi$ and $\mu$ in $\Delta_{\xi \mu}$, and by comparing with the explicit result for $T^{S}$ in Eq. (42), it is very simple to show that the identity of Eq. (11) is also preserved.

Now we consider the identity given in Eq. (10), involving the amplitudes $T_{\mu \nu}^{A A}, T_{\mu}^{A P}$, and $T_{\mu}^{V}$. Contracting $T_{\mu \nu}^{A A}$ given in Eq. (50) with $q^{\mu}$, we get

$$
\begin{aligned}
q^{\mu} T_{\mu \nu}^{A A}\left(k_{1}, k_{2}\right)= & 2 M\left\{-4 M q_{\nu}\left[I_{\log }\left(M^{2}\right)\right.\right. \\
& \left.\left.-i(4 \pi)^{-2} Z_{0}\left(q^{2} ; M^{2}\right)\right]\right\}+q^{\mu} A_{\mu \nu} .
\end{aligned}
$$

It is easy to verify that using Eqs. (43) and (46), the identity in Eq. (10) is preserved.

We proceed by examining the relation given in Eq. (12) using Eq. (46) for the amplitude $T_{\mu}^{A P}\left(k_{1}, k_{2}\right)$. Adding and subtracting scalar one-point functions carrying momenta $k_{1}$ and $k_{2}$, one can easily check that this expression for $T^{P P}\left(k_{1}, k_{2}\right)$ naturally leads to preservation of the relation in Eq. (12). Also, the relations involving the $A V$ two-point function in Eqs. (13) and (14) are immediate: the Green's functions $T_{\mu}^{P V}$ and $T_{\mu}^{A}$ on the r.h.s. of Eqs. (13) and (14) are identically zero due to properties the Dirac traces, and on the left-hand side one also obtains a zero because the contractions of the explicit expression for $T_{\mu \nu}^{A V}\left(k_{1}, k_{2}\right)$ in Eq. (48) with $\left(k_{1}-k_{2}\right)^{\mu}$ or $\left(k_{1}-k_{2}\right)^{\mu}$ are zero due to the antisymmetry of $\varepsilon_{\mu \nu \lambda \xi}$.

Next, we turn our attention to the tensorial amplitudes. We start with the relations of Eqs. (20) and (25). Initially we note that the amplitude $T_{\alpha \mu \nu}^{V T}\left(k_{1}, k_{2}\right)$ given in Eq. (54) can be reorganized as

$$
\begin{aligned}
T_{\alpha \mu \nu}^{V T}\left(k_{1}, k_{2}\right)= & \frac{1}{2 M} q_{\mu}\left[T_{\alpha \nu}^{V V}\left(k_{1}, k_{2}\right)-T_{\alpha \nu}^{A A}\left(k_{1}, k_{2}\right)\right] \\
& -\frac{1}{2 M} q_{\nu}\left[T_{\alpha \mu}^{V V}\left(k_{1}, k_{2}\right)-T_{\alpha \mu}^{A A}\left(k_{1}, k_{2}\right)\right],
\end{aligned}
$$

where we have used Eqs. (49) and (50). Written in this form, it is now trivial to see that Eqs. (20) and (25) are satisfied. Next, we consider the relation in Eq. (18). The $T T$ two-point function, given by Eq. (55), after some algebraic effort can be put in the form 


$$
\begin{aligned}
T_{\alpha \beta \mu \nu}^{T T}\left(k_{1}, k_{2}\right)= & g_{\alpha \mu} T_{\beta \nu}^{A A}\left(k_{1}, k_{2}\right)-g_{\alpha \nu} T_{\beta \mu}^{A A}\left(k_{1}, k_{2}\right) \\
& +g_{\beta \nu} T_{\alpha \mu}^{A A}\left(k_{1}, k_{2}\right)-g_{\beta \mu} T_{\alpha \nu}^{A A}\left(k_{1}, k_{2}\right) \\
& +\left(g_{\alpha \mu} g_{\beta \nu}-g_{\alpha \nu} g_{\beta \mu}\right) T^{S S}\left(k_{1}, k_{2}\right),
\end{aligned}
$$

where the Eqs. (44) and (50) have been used. It is now evident that the contraction with the external momentum leads to Eq. (18). Now, we consider the constraint given in Eq. (24). First, we note that Eq. (51) for the $S T$ amplitude, by using Eqs. (15) and (47), can be rewritten as

$$
\begin{aligned}
T_{\mu \nu}^{S T}\left(k_{1}, k_{2}\right) & =\frac{1}{2 M}\left[q_{\mu} T_{\nu}^{S V}\left(k_{1}, k_{2}\right)-q_{\nu} T_{\mu}^{S V}\left(k_{1}, k_{2}\right)\right], \\
& =-\frac{i}{2} \varepsilon_{\mu \nu \lambda \xi}\left(T^{A V}\right)^{\lambda \xi}\left(k_{1}, k_{2}\right) .
\end{aligned}
$$

Therefore, contraction with the external momentum

$$
\begin{aligned}
q^{\mu} T_{\mu \nu}^{T S}\left(k_{1}, k_{2}\right) & =\frac{1}{2 M}\left\{q^{2} T_{\nu}^{S V}\left(k_{1}, k_{2}\right)-q_{\nu}\left[T^{S}\left(k_{2}\right)-T^{S}\left(k_{1}\right)\right]\right\} \\
& =-\frac{i}{2} \varepsilon_{\mu \nu \lambda \xi} q^{\mu}\left(T^{A V}\right)^{\lambda \xi}\left(k_{1}, k_{2}\right),
\end{aligned}
$$

leads immediately to Eq. (24). Now, noting that by Eqs. (48) and (52) the $A V$ Green's function is identical to the $T P$ function, we have that

$$
q^{\mu} T_{\mu \nu}^{T P}\left(k_{1}, k_{2}\right)=-2 M T_{\nu}^{V P}\left(k_{1}, k_{2}\right),
$$

as it should to satisfy Eq. (21). Also, comparing Eqs. (47) and (53), one sees that

$$
T_{\alpha \mu \nu}^{A T}\left(k_{1}, k_{2}\right)=-i \varepsilon_{\mu \nu \alpha \beta}\left(T^{S V}\right)^{\beta}\left(k_{1}, k_{2}\right),
$$

and then

$$
\begin{gathered}
q^{\alpha} T_{\mu \nu \alpha}^{T A}\left(k_{1}, k_{2}\right)=2 M T_{\mu \nu}^{T P}\left(k_{1}, k_{2}\right), \\
q^{\mu} T_{\mu \nu \alpha}^{T A}\left(k_{1}, k_{2}\right)=-2 M T_{\nu \alpha}^{V A}\left(k_{1}, k_{2}\right) \\
=-i \varepsilon_{\nu \alpha \lambda \xi} q^{\lambda}\left(T^{S V}\right)^{\xi}\left(k_{1}, k_{2}\right) .
\end{gathered}
$$

Therefore, the remaining relations, given by Eqs. (22) and (23), are also satisfied.

This completes the verification of the consistency of the manipulations performed at the one-loop level. It is important to emphasize that all the relations among Green's functions which can be stated at the level of integrands are preserved, in spite of the presence of ambiguous terms. The arbitrariness concerning the choice of the regularization is also preserved since only very general mathematical properties have been assumed.

The crucial point now is that the preservation of the relations among Green's functions is not the only requirement one should ask for a consistent regularization scheme since, as we have seen, the amplitudes themselves contain ambiguities that are functions of the ambiguous combination of momenta $Q=k_{1}+k_{2}$. Therefore, one must have a scheme that ensures elimination of the ambiguous terms from the amplitudes themselves, not only in the relations involving two or more of them. In the next section, we discuss the constraints imposed by symmetry relations (Furry's theorem and Ward identities) on special amplitudes and they will provide guidance for dealing with the ambiguous terms.

\section{AMBIGUITIES AND PRESERVATION OF SYMMETRIES}

In the process of constructing a consistent interpretation for the divergent one-loop amplitudes the preservation of symmetries plays a central role. In principle, there is no a priori reason for expecting that space-time symmetries will be automatically manifest in divergent amplitudes. However, it seems nevertheless reasonable to expect that one should be able to identify general properties that the divergent quantities must satisfy in order to guarantee the preservation of such fundamental symmetries. In this sense, the regularization method itself is not the most important ingredient, what really matters are the requirements that quantities like $\nabla_{\beta \mu}, \square_{\alpha \beta \mu \nu}$, and $\Delta_{\alpha \beta}$ must obey to preserve the symmetry relations. Having this in mind, let us now consider the symmetry properties pertinent to the one- and two-point amplitudes we are discussing. We shall refer to the Ward identities and other general constraints imposed by Furry's theorem on these amplitudes.

We start considering the simplest amplitude that carries one Lorentz vector index, the amplitude $T_{\mu}^{V}\left(k_{1}\right)$. On general symmetry grounds, Furry's theorem states that this amplitude must be zero. So, from Eq. (43), Furry's theorem requires that

$$
\begin{aligned}
T_{\mu}^{V}= & -k_{1}^{\beta} \nabla_{\beta \mu}-\frac{1}{3} k_{1}^{\beta} k_{1}^{\alpha} k_{1}^{\nu}\left[\square_{\alpha \beta \mu \nu}\right]+\frac{1}{3} k_{1}^{2} k_{1}^{\nu}\left[\Delta_{\nu \mu}\right] \\
& +\frac{2}{3} k_{1 \mu} k_{1}^{\alpha} k_{1}^{\beta}\left[\Delta_{\alpha \beta}\right]=0 .
\end{aligned}
$$

There are two different ways to satisfy this requirement. The first one is the choice $k_{1}=0$. But, is it always possible to make this choice? Thinking on $T_{\mu}^{V}$ in isolation, the answer to this question is affirmative, since $k_{1}$ is arbitrary. However, $T_{\mu}^{V}$ is not the only amplitude in the theory and so one must ask the question if this choice is not invalidating other symmetry relations. For example, the relation given in Eq. (8) relates this amplitude to $\left(k_{1}-k_{2}\right)^{\mu} T_{\mu \nu}^{V V}\left(k_{1}, k_{2}\right)$, which we repeat here for clarity, is given by

$$
\left(k_{1}-k_{2}\right)^{\mu} T_{\mu \nu}^{V V}\left(k_{1}, k_{2}\right)=T_{\nu}^{V}\left(k_{1}\right)-T_{\nu}^{V}\left(k_{2}\right) .
$$

Vector current conservation demands that $\left(k_{1}-\right.$ $\left.k_{2}\right)^{\mu} T_{\mu \nu}^{V V}\left(k_{1}, k_{2}\right)=0$. Therefore, the difference of the two one-point functions on the r.h.s. of Eq. (85) having dependencies on $k_{1}$ and $k_{2}$ needs to be zero. Obviously, the simultaneous choice $k_{1}=0$ and $k_{2}=0$, which would satisfy both requirements, cannot be made because this would imply $q=k_{1}-k_{2}=0$ always. Therefore, we need another way to satisfy Eq. (84). Since the requirement of Eq. (84) involves the divergent quantities $\square_{\alpha \beta \mu \nu}, \nabla_{\mu \nu}$, 
and $\Delta_{\mu \nu}$, one could ask for a regularization scheme that leads to

$$
\square_{\alpha \beta \mu \nu}^{\mathrm{reg}}=\nabla_{\mu \nu}^{\mathrm{reg}}=\Delta_{\mu \nu}^{\mathrm{reg}}=0,
$$

where the superscript "reg" means that the integrals defining these quantities are regularized.

The same conclusion is reached considering the explicit expression for $T_{\mu \nu}^{V V}\left(k_{1}, k_{2}\right)$ given in Eq. (49). Contracting it with the external momentum $q^{\mu}=\left(k_{1}-k_{2}\right)^{\mu}$, one obtains

$$
\begin{aligned}
q^{\mu} T_{\mu \nu}^{V V}\left(k_{1}, k_{2}\right)= & 4\left\{q^{\alpha}\left[\nabla_{\alpha \nu}\right]\right. \\
& +\left(k_{1}^{\alpha} k_{1}^{\beta} k_{1}^{\rho}-k_{2}^{\alpha} k_{2}^{\beta} k_{2}^{\rho}\right) \frac{1}{3}\left[\square_{\alpha \beta \rho \nu}\right] \\
& -\left(k_{1}^{2} k_{1}^{\rho}-k_{2}^{2} k_{2}^{\rho}\right) \frac{1}{3}\left[\Delta_{\rho \nu}\right] \\
& \left.-\left(k_{1 \nu} k_{1}^{\alpha} k_{1}^{\beta}-k_{2 \nu} k_{2}^{\alpha} k_{2}^{\beta}\right) \frac{2}{3}\left[\Delta_{\alpha \beta}\right]\right\} .
\end{aligned}
$$

Since a conserved vector current should not be obtained by convenient choices of the arbitrary momenta $k_{1}$ and $k_{2}$, the conditions of Eq. (86) seem therefore also necessary here.

For the same reason that $T_{\mu}^{V}\left(k_{1}\right)$ must vanish, other vector two-point functions need to vanish identically. These are $T_{\mu}^{V S}\left(k_{1}, k_{2}\right)$ and $T_{\mu \nu}^{A V}\left(k_{1}, k_{2}\right)$ which, from Eqs. (47) and (48), imply in

$$
\begin{gathered}
Q^{\xi}\left[\Delta_{\mu \xi}\right]=0, \\
\varepsilon_{\mu \nu \alpha \beta} q^{\beta} Q_{\xi}\left[\Delta^{\xi \alpha}\right]=0 .
\end{gathered}
$$

In principle, for these two specific amplitudes both options, of choosing $k_{1}$ and $k_{2}$ in a convenient way or constructing $\Delta_{\mu \beta}^{\mathrm{reg}}=0$, are possible. For example, considering the contractions of these amplitudes with external momenta we obtain

$$
\begin{gathered}
q^{\mu} T_{\mu}^{V S}\left(k_{1}, k_{2}\right)=-4 M q^{\mu} Q^{\beta}\left[\Delta_{\mu \beta}\right], \\
q^{\mu} T_{\mu \nu}^{A V}\left(k_{1}, k_{2}\right)=-2 \varepsilon_{\mu \nu \alpha \beta} q^{\mu} q^{\beta} Q_{\xi}\left[\Delta^{\xi \alpha}\right], \\
q^{\nu} T_{\mu \nu}^{A V}\left(k_{1}, k_{2}\right)=-2 \varepsilon_{\mu \nu \alpha \beta} q^{\nu} q^{\beta} Q_{\xi}\left[\Delta^{\xi \alpha}\right] .
\end{gathered}
$$

A conserved vector current for $T_{\mu}^{V S}\left(k_{1}, k_{2}\right)$ can be obtained with the choice $k_{1}=-k_{2}$, or by taking $\Delta_{\mu \nu}^{\mathrm{reg}}=0$. However, both contractions involving $T_{\mu \nu}^{A V}\left(k_{1}, k_{2}\right)$ vanish identically independently of the two possible choices, just because the antisymmetric $\varepsilon_{\mu \nu \alpha \beta}$ is contracted with a symmetric object. The vector current must be conserved, but the axial-vector current must not. So there is only one consistent value for $T_{\mu \nu}^{A V}\left(k_{1}, k_{2}\right)$ : the identically zero value. Otherwise, a symmetry relation is broken. We can add to this argumentation another very general aspect that forces us to obtain a zero value for $T_{\mu \nu}^{A V}\left(k_{1}, k_{2}\right)$ (and $T_{\mu}^{V S}$ ): unitarity. If the amplitude does not vanish, then it needs to develop an imaginary part at $q^{2}=4 M^{2}$ to be consistent with unitarity (Cutkosky's rules). Clearly, from Eqs. (47) and (48) for $T_{\mu}^{V S}\left(k_{1}, k_{2}\right)$ and $T_{\mu \nu}^{A V}\left(k_{1}, k_{2}\right)$, respectively, this cannot happen.

Next, we consider $T_{\mu \nu}^{A A}\left(k_{1}, k_{2}\right)$. Using its explicit expression given in Eq. (50), one can show that

$$
\begin{aligned}
q^{\mu} T_{\mu \nu}^{A A}\left(k_{1}, k_{2}\right)= & 4\left\{-q^{\alpha}\left[\nabla_{\alpha \nu}\right]\right. \\
& +\left(k_{1}^{\alpha} k_{1}^{\beta} k_{1}^{\rho}-k_{2}^{\alpha} k_{2}^{\beta} k_{2}^{\rho}\right) \frac{1}{3}\left[\square_{\alpha \beta \rho \nu}\right] \\
& +\left(k_{1}^{2} k_{1}^{\rho}-k_{2}^{2} k_{2}^{\rho}\right) \frac{1}{3}\left[\Delta_{\rho \nu}\right] \\
& \left.+\left(k_{1 \nu} k_{1}^{\alpha} k_{1}^{\beta}-k_{2 \nu} k_{2}^{\alpha} k_{2}^{\beta}\right) \frac{2}{3}\left[\Delta_{\alpha \beta}\right]\right\} \\
& -2 M i T_{\nu}^{P A}\left(k_{1}, k_{2}\right) .
\end{aligned}
$$

However, the proportionality between the axial vector and the pseudoscalar current states that $q^{\mu} T_{\mu \nu}^{A A}\left(k_{1}, k_{2}\right)=$ $-2 \operatorname{MiT}_{\nu}^{P A}\left(k_{1}, k_{2}\right)$. Therefore, one arrives at the same conclusion as for the amplitude $T_{\mu \nu}^{V V}\left(k_{1}, k_{2}\right)$, that the relations given in Eq. (86) must be satisfied, since $T_{\nu}^{P A}\left(k_{1}, k_{2}\right)$ is free from ambiguities, see Eq. (46). Also, the same conclusion is obtained if one rewrites $q^{\mu} T_{\mu \nu}^{A A}$ in terms of the amplitudes $T_{\nu}^{V}\left(k_{1}\right)$, as in Eq. (10).

Considering all amplitudes and their symmetry relations, the same conditions will emerge: there is no consistent interpretation for the one-loop divergent amplitudes if the conditions given in Eq. (86), that we call CR's, are not fulfilled. In principle, one could argue that the imposition of the CR's represents an arbitrary choice, which is at the same level of the choice of a specific regulating distribution to be used in the integrands of the divergent integrals. However, this is not true. One should consider the CR's as a fundamental requirement to be imposed on the one-loop divergent amplitudes in order to materialize the fundamental space-time symmetries. Any calculation that violates the CR's has the potential of predicting unphysical results, since it leads to the destruction of the foundations of the theory which have generated the amplitudes themselves. Therefore, the CR's do not represent arbitrary choices, because there is no option out of these properties capable to allow a consistent interpretation of the calculations. Note that the CR's not only remove all the ambiguous terms, which are always symmetry violating, but also remove all the symmetry-violating terms, which are not always ambiguous.

To finalize this section, we summarize results by defining what we denominate the "consistently regularized amplitudes" denoted by $\mathcal{T}^{S}, \mathcal{T}_{\mu}^{V S}, \mathcal{T}^{S S}, \cdots$. These are, respectively, the amplitudes $\mathcal{T}^{S}, \mathcal{T}_{\mu}^{V S}, \mathcal{T}^{S S}, \cdots$ obtained previously, with the terms containing the pieces proportional to the quantities $\square_{\alpha \beta \mu \nu}, \nabla_{\mu \nu}$, and $\Delta_{\mu \nu}$ removed, as demanded by the CR's given in Eq. (86). Explicitly, they are given by

(I) One-point functions:

$$
\begin{gathered}
\mathcal{T}^{S}=4 M\left[I_{\text {quad }}\left(M^{2}\right)\right], \\
\mathcal{T}_{\mu}^{V}=0 .
\end{gathered}
$$


(II) Two-point functions:

$$
\begin{aligned}
\mathcal{T}_{\mu}^{V S}(q)=\mathcal{T}_{\mu \nu}^{A V}(q)=0, & \\
\mathcal{T}^{S S}(q)=4 & \left\{\left[I_{\text {quad }}\left(M^{2}\right)\right]+\frac{1}{2}\left(4 M^{2}-q^{2}\right)\left[I_{\log }\left(M^{2}\right)\right]\right. \\
- & \left.\frac{1}{2}\left(4 M^{2}-q^{2}\right)\left(\frac{i}{16 \pi^{2}}\right)\left[Z_{0}\left(q^{2}, M^{2}\right)\right]\right\}, \\
\mathcal{T}^{P P}(q)= & 4\left\{-\left[I_{\text {quad }}\left(M^{2}\right)\right]+\frac{1}{2} q^{2}\left[I_{\log }\left(M^{2}\right)\right]\right. \\
& \left.-\frac{1}{2} q^{2}\left(\frac{i}{16 \pi^{2}}\right)\left[Z_{0}\left(q^{2}, M^{2}\right)\right]\right\}, \\
\mathcal{T}_{\mu}^{P A}(q)= & 4 M q_{\mu}\left\{\left[I_{\log }\left(M^{2}\right)\right]-\left(\frac{i}{16 \pi^{2}}\right)\right. \\
& \left.\times\left[Z_{0}\left(q^{2}, M^{2}\right)\right]\right\}, \\
\mathcal{T}_{\mu \nu}^{V V}(q)= & \frac{4}{3}\left(q^{2} g_{\mu \nu}-q_{\mu} q_{\nu}\right)\left\{\left[I_{\log }\left(M^{2}\right)\right]\right. \\
& -\left(\frac{i}{16 \pi^{2}}\right)\left[\frac{1}{3}+\frac{\left(2 M^{2}+q^{2}\right)}{q^{2}}\right. \\
& \left.\left.\times\left[Z_{0}\left(q^{2}, M^{2}\right)\right]\right]\right\},
\end{aligned}
$$

$$
\begin{aligned}
\mathcal{T}_{\mu \nu}^{A A}(q)= & \frac{4}{3}\left(q^{2} q_{\mu \nu}-q_{\mu} q_{\nu}\right)\left\{\left[I_{\log }\left(M^{2}\right)\right]-\left(\frac{i}{16 \pi^{2}}\right)\right. \\
& \left.\times\left[\frac{1}{3}+\frac{\left(2 M^{2}+q^{2}\right)}{q^{2}}\left[Z_{0}\left(q^{2}, M^{2}\right)\right]\right]\right\} \\
& -8 M^{2} g_{\mu \nu}\left\{\left[I_{\log }\left(M^{2}\right)\right]-\left(\frac{i}{16 \pi^{2}}\right)\right. \\
& \left.\times\left[Z_{0}\left(q^{2}, M^{2}\right)\right]\right\} .
\end{aligned}
$$

The tensor amplitudes can be written as

$$
\begin{gathered}
\mathcal{T}_{\mu \nu}^{S T}(q)=\mathcal{T}_{\mu \nu}^{P T}(q)=\mathcal{T}_{\alpha \mu \nu}^{A T}(q)=0, \\
\mathcal{T}_{\alpha \mu \nu}^{V T}(q)=4 M\left(g_{\alpha \nu} g_{\mu \lambda}-g_{\alpha \mu} g_{\nu \lambda}\right) q^{\lambda}\left\{\left[I_{\log }\left(M^{2}\right)\right]\right. \\
\left.-\left(\frac{i}{16 \pi^{2}}\right)\left[Z_{0}\left(q^{2}, M^{2}\right)\right]\right\},
\end{gathered}
$$

\section{PHENOMENOLOGY AND NUMERICAL RESULTS}

The amplitudes obtained in the preceding section are free of ambiguities and are symmetry preserving. In this paper, we have focused on one- and two-point functions, where resides the highest degree of divergence, however similar results can be obtained for three- and four-point functions used in the model to describe meson decays and meson-meson interactions. All these mathematical structures also appear in fundamental theories. Here, as a consequence of the adopted strategy to handle the divergent structures, it must be noted that such structures are treated in a very closely related way as in renormalization procedures of renormalizable theories. In the obtained expressions for the calculated amplitudes, only two divergent objects have survived after the adoption of CR's, namely $I_{\log }\left(M^{2}\right)$ and $I_{\text {quad }}\left(M^{2}\right)$. The next step, if the amplitudes were to be considered in the context of fundamental theo- ries, is the elimination of such objects through the reparametrization of the theory in the renormalization of physical parameters. Since $I_{\log }\left(M^{2}\right)$ and $I_{\text {quad }}\left(M^{2}\right)$ are completely absorbed in this process, the regularization eventually used plays no relevant role, due to the fact that the renormalized expressions are independent of particular aspects of the chosen regularization. The theory is predictive, given the fact that the results for the amplitudes associated to physical processes do not depend on the choices involved in the intermediary steps.

In the case of the NJL model, considering the nonrenormalizable character, the remaining undefined objects need to be specified in some way by using physical parameters chosen as inputs of the model. The traditional way to make predictions in the context of NJL involves regularization such as the introduction of a 3D- or 4D-cutoff $\Lambda$ in the involved Feynman integrals. The expressions for the amplitudes are, in this way, dependent on the parameters of the chosen regularization distribution as well as, if the CR's 
are not satisfied, on the chosen routing for internal lines momenta. In this scenario, the model contains at least two parameters (in the chiral limit $m_{0}=0$ ); the coupling strength $G_{S}$ and a regularization parameter (the cutoff $\Lambda$ ). The constituent quark mass $M$ is not an input parameter as it is given by the solution of the gap equation. The parameters $G_{S}$ and $\Lambda$ need to be fixed through two items of experimental information. The quark condensate $\langle\bar{\psi} \psi\rangle$ and the pion-decay constant $f_{\pi}$ are usually used for this purpose. The first is related to a quadratically divergent amplitude while the second is related to a logarithmically divergent one. The values for $G_{S}$ and $\Lambda$ which give the best adjustments to the experimental values of $\langle\bar{\psi} \psi\rangle$ and $f_{\pi}$ will depend on the specific form of the regularization distribution. As a consequence, all the physical amplitudes describing processes pertinent to the model are affected by the choice of the regularization. Because of this reason, the chosen regularization must be part of the model. The predictive power of the original quantum field model is affected since the predictions are dependent on a choice which characterizes an ambiguity. This is not the desirable situation. We wish any model prediction becomes unique in a similar way as it happens in renormalizable theories. The regularization must become just a convenient choice in the intermediary steps. In order to show that it is possible to achieve the desirable situation referred above, we first note that the adoption of the consistently regularized amplitudes listed in the preceding section implies that we can only adopt regularizations that fulfill the CR's. This does not represent a choice as, following our analysis, regularizations which break the CR's will lead to ambiguities as well as to symmetry violations, and this is unacceptable. If we want a predictive model, only regularizations preserving the CR's make sense. After these important remarks, let us now show how the arbitrariness associated to the choice of regularization can be completely removed.

Having this in mind, we start by showing how to relate the remaining objects $I_{\log }\left(M^{2}\right)$ and $I_{\text {quad }}\left(M^{2}\right)$ to physical observables chosen as input of the model. First, we point out that the quark condensate $\langle\bar{\psi} \psi\rangle$ is related to the $\mathcal{T}^{S}$ one-point function as

$$
\langle\bar{\psi} \psi\rangle=-N_{c} \mathcal{T}^{S} .
$$

Substituting the result (94) we get

$$
\langle\bar{\psi} \psi\rangle=-4 N_{c} M\left[i I_{\text {quad }}\left(M^{2}\right)\right] .
$$

For the second, we note that, in the context of the NJL model, mesons are relativistic quark-antiquark bound states. In the random-phase approximation, the meson propagators can be written as (see, for example, Ref. [5])

$$
D_{\mathcal{M}}\left(q^{2}\right)=\frac{2 G_{S}}{1-2 G_{S} \Pi_{\mathcal{M}}\left(q^{2}\right)},
$$

where $\Pi_{\mathcal{M}}$ is the polarization function defined by

$$
\Pi_{\mathcal{M}}\left(q^{2}\right)=i \int \frac{d^{4} k}{(2 \pi)^{4}} \operatorname{Tr}\left\{\Gamma_{\mathcal{M}} S\left(k+k_{1}\right) \Gamma_{\mathcal{M}} S\left(k+k_{2}\right)\right\},
$$

with $S$ being the quark propagator defined previously. $\Gamma_{\mathcal{M}}$ stands for the flavor and Dirac matrices giving the quantum numbers of the meson $\mathcal{M}$. For example, for the neutral pion, $\Gamma_{\mathcal{M}}=\tau_{3} \gamma_{5}$, for the scalar-isoscalar meson, $\Gamma_{\mathcal{M}}=$ 1 . In writing the equations above, we assumed the most general labels for the momenta $k_{1}$ and $k_{2}$ running in the internal lines of the loop integral. The physical momentum $q$ is defined as the difference $k_{1}-k_{2}$ as imposed by energy-momentum conservation at each vertex.

The pole of the propagator in Eq. (107), calculated at $q^{2}=m_{\mathcal{M}}^{2}$, gives the mass of the respective meson. The condition for the pion mass is given by

$$
1-2 G_{S} \Pi_{\pi}\left(m_{\pi}^{2}\right)=0,
$$

where

$$
\Pi_{\pi}\left(q^{2}\right)=-i N_{c} N_{f}\left[\mathcal{T}^{P P}(q)\right] .
$$

Substituting now the explicit form of $\mathcal{T}^{P P}(q)$, derived previously and given in Eq. (98), we obtain

$$
\begin{aligned}
\Pi_{\pi}\left(q^{2}\right)= & 4 i N_{c} N_{f}\left[I_{\text {quad }}\left(M^{2}\right)\right]-2 i N_{c} N_{f} q^{2}\left\{\left[I_{\log }\left(M^{2}\right)\right]\right. \\
& \left.-\left(\frac{i}{16 \pi^{2}}\right)\left[Z_{0}\left(q^{2}, M^{2}\right)\right]\right\} .
\end{aligned}
$$

Using the Eq. (3) in order to eliminate $I_{\text {quad }}\left(M^{2}\right)$ and evaluating Eq. (111) at $q^{2}=m_{\pi}^{2}$, we get the following expression for the pion mass:

$$
m_{\pi}^{2}=i \frac{m_{0}}{4 N_{c} N_{f} M G_{S}} \frac{1}{\left\{i\left[I_{\mathrm{log}}\left(M^{2}\right)\right]+\left(\frac{1}{16 \pi^{2}}\right)\left[Z_{0}\left(m_{\pi}^{2}, M^{2}\right)\right]\right\}} .
$$

As seen in the chiral limit $\left(m_{0}=0\right)$, the pion becomes massless $\left(m_{\pi}=0\right)$, in agreement with Goldstone's theorem.

The pion phenomenology is also characterized by the decay constant $f_{\pi}$. Experimentally it is related to the weak decay $\pi^{ \pm} \rightarrow \mu^{ \pm}+\nu_{\mu}$ and is calculated from the vacuum to the one-pion axial-vector current matrix element

$$
\left\langle 0\left|\bar{\psi}(x) \gamma_{\mu} \gamma_{5} \tau^{i} / 2 \psi(x)\right| \pi^{j}(q)\right\rangle=i f_{\pi} q_{\mu} \delta_{i j} e^{-i q x},
$$

where $\left|\pi^{j}(q)\right\rangle$ is a pion state with four-momentum $q$. At one-loop order, one can express this matrix element in terms of the $\mathcal{T}_{\mu}^{A P}$ two-point function as

$$
i f_{\pi} q_{\mu} \delta_{i j}=-N_{c} g_{\pi q q} \delta_{i j}\left[\mathcal{T}_{\mu}{ }^{A P}(q)\right],
$$

where $g_{\pi q q}$ is the pion-to-quark-quark coupling strength, related to the residue of Eq. (107) as

$$
g_{\pi q q}^{2}=\left.\left(\frac{\partial \Pi_{P P}\left(q^{2}\right)}{\partial q^{2}}\right)^{-1}\right|_{q^{2}=m_{\pi}^{2}} .
$$


Using Eqs. (99) and (111), we can write

$$
f_{\pi}=-4 N_{c} g_{\pi q q} M\left\{i\left[I_{\log }\left(M^{2}\right)\right]+\left(\frac{1}{16 \pi^{2}}\right)\left[Z_{0}\left(m_{\pi}^{2}, M^{2}\right)\right]\right\},
$$

$$
\begin{aligned}
g_{\pi q q}^{-2}= & -2 N_{c} N_{f}\left\{i\left[I_{\log }\left(M^{2}\right)\right]+\left(\frac{1}{16 \pi^{2}}\right)\left[Z_{0}\left(m_{\pi}^{2}, M^{2}\right)\right]\right\} \\
& -2 N_{c} N_{f} m_{\pi}^{2}\left(\frac{1}{16 \pi^{2}}\right)\left[Y_{1}\left(m_{\pi}^{2}, M^{2}\right)\right],
\end{aligned}
$$

where $Y_{1}\left(q^{2}, M^{2}\right)$ is the $k=1$ element of the set

$$
Y_{k}\left(q^{2} ; m^{2}\right)=\int_{0}^{1} d z \frac{z^{k}(1-z)}{q^{2} z(1-z)-m^{2}} .
$$

In a completely similar way, for the scalar meson $(\sigma)$ we have

$$
\begin{aligned}
m_{\sigma}^{2}= & 4 M^{2}-\frac{m_{0}}{M} \\
& \times \frac{1}{4 G_{S} N_{c} N_{f}\left\{i I_{\log }\left(M^{2}\right)+\left(\frac{1}{16 \pi^{2}}\right)\left[Z_{0}\left(m_{\sigma}^{2}, M^{2}\right)\right]\right\}}, \\
g_{\sigma q q}^{-2}= & 2 i N_{c} N_{f}\left(m_{\sigma}^{2}-4 M^{2}\right)\left(\frac{i}{16 \pi^{2}}\right)\left[Y_{1}\left(m_{\sigma}^{2}, M^{2}\right)\right] \\
& -2 i N_{c} N_{f}\left\{I_{\log }\left(M^{2}\right)-\left(\frac{i}{16 \pi^{-2}}\right)\left[Z_{0}\left(m_{\sigma}^{2}, M^{2}\right)\right]\right\} .
\end{aligned}
$$

It is easy to see from Eqs. (116) and (117) that the observable $f_{\pi}$ can be related to the undefined quantity $I_{\log }$. In order to make the aspects we want to emphasize clear, we initially consider this relation in the chirally symmetric case. Then we get

$$
i I_{\log }\left(M^{2}\right)=-\frac{f_{\pi}^{2}}{2 N_{c} N_{f} M^{2}},
$$

since $Z_{0}\left(m_{\pi}^{2}=0\right)=0$. Through the Eqs. (106) and (121), we have stated relations between two observables (inputs), the quark condensate and the pion-decay constant, with two undefined objects coming from loop calculations. These objects are functions of the constituent quark mass which must be determined in some stage. Therefore the Eqs. (106) and (121) in fact represent the relation of two quantities to two functions. If we want to know such functions, we have to integrate $I_{\log }\left(M^{2}\right)$ and $I_{\text {quad }}\left(M^{2}\right)$ which means to adopt an explicit form of regularization distribution. This process introduces at least one regularization parameter $\Lambda$ as it is well known. Different regularizations will generally lead to different values of $\Lambda$ as it is usual in the context of the NJL model with regularizations. In order to avoid this situation, we will proceed in a different way.
First, we note that those two functions are not independent. It is possible to show that they are related by

$$
\frac{\partial}{\partial M^{2}}\left[i I_{\text {quad }}\left(M^{2}\right)\right]=i I_{\log }\left(M^{2}\right) \text {. }
$$

On the other hand, we can also state that $I_{\log }\left(M^{2}\right)$ possesses the following property:

$$
\frac{\partial}{\partial M^{2}}\left[i I_{\log }\left(M^{2}\right)\right]=\frac{1}{16 \pi^{2} M^{2}} .
$$

In order to satisfy these two conditions, it is necessary to get the following general forms:

$$
\begin{gathered}
i I_{\log }\left(M^{2}\right)=\frac{1}{16 \pi^{2}} \ln M^{2}+C_{1}, \\
i I_{\text {quad }}\left(M^{2}\right)=\frac{1}{16 \pi^{2}} M^{2}\left[\ln M^{2}-1+C_{1}\right]+C_{2},
\end{gathered}
$$

where $C_{1}$ and $C_{2}$ are indeterminate constants $-C_{1}$ is dimensionless and $C_{2}$ has dimension of (mass) ${ }^{2}$. In the context of regularizations, $C_{1}$ and $C_{2}$ are related to the regularization parameter $\Lambda$. Eliminating the constant $C_{1}$ we see that

$$
i I_{\text {quad }}\left(M^{2}\right)=\frac{-1}{16 \pi^{2}} M^{2}+M^{2}\left[i I_{\log }\left(M^{2}\right)\right]+C_{2} .
$$

Replacing $I_{\text {quad }}$ and $I_{\log }$ in terms of $\langle\bar{\psi} \psi\rangle$ and $f_{\pi}$, Eqs. (106) and (121), we get

$$
\frac{M^{3}}{16 \pi^{2}}+\left(\frac{f_{\pi}^{2}}{2 N_{c} N_{f}}-C_{2}\right) M-\frac{\langle\bar{\psi} \psi\rangle}{4 N_{c}}=0 .
$$

There are two important aspects involved in the above equation. First, it ensures that it is crucial to obey the properties (122) and (123) when the functions $I_{\log }\left(M^{2}\right)$ and $I_{\text {quad }}\left(M^{2}\right)$ are made explicit. These two properties work as additional constraints to be required of a regularization distribution if one wants to get consistency in perturbative calculations. The violation of these properties will result in breaking the scale properties of physical amplitudes, and possesses the same status of symmetry violations [25]. The second aspect refers to the dependence of the physical parameters on the choice of the specific regularization. The above expression states that even if a regularization obeys the CR's and simultaneously the conditions (122) and (123) it remains a freedom to distinguish it from other regularizations belonging to the class of consistent regularizations, which is the value of $C_{2}$. Two consistent regularizations can differ only by the value of $C_{2}$. As a consequence, the physical implications of the model seem to be definitely regularization dependent. The introduction of experimental values for the inputs $\langle\bar{\psi} \psi\rangle$ and $f_{\pi}$ makes it necessary to specify $C_{2}$ to get $M$ and then through the gap equation to get the value for $G_{S}$. There is nothing more to be imposed, based on consistency reasons, to remove this arbitrariness. Apparently all our 
efforts cannot avoid the dependence of the results on the choice of the regularization, even if we have drastically restricted the regularizations which can be used in the calculations.

At this point it seems there is nothing else to do except to choose convenient values for $C_{2}$. The convenience of such choices is related to the fact that we need to choose an adequate value for $C_{2}$ in order to get a good value for $M$ and after this to find a good value for $G_{S}$. It is precisely in this process that emerges the most surprising aspect of our formulation. If we recognize that only positive values of $M$ make sense in the equation, the nonlinear character of the equation in $M$ produces a critical condition to possible values of $C_{2}$. It turns out that one finds solutions with $M>$ 0 only for $C_{2} \geq C_{\text {crit }}$, such that

$$
\begin{gathered}
C_{2}<C_{\text {crit }} \rightarrow \text { no solutions } \quad C_{2}=C_{\text {crit }} \rightarrow \text { one solution } \\
C_{2}>C_{\text {crit }} \rightarrow \text { two solutions. }
\end{gathered}
$$

Therefore, it seems obvious that there is only one value for $C_{2}$ which is reasonable, the $C_{\text {crit }}$, due to the fact that only this value allows us a consistent physical interpretation of the model predictions. Assuming this attitude, the NJL model becomes predictive since the remaining arbitrariness is fixed through the existence of a critical condition. There are considerable differences between our formulation and the traditional ones. The first and immediate refers to the determination of the constituent quark mass $M$. Such value is fixed by the critical point in the diagram $M \times C_{2}$. The value of the mass, therefore, depends only on the experimental values for $\langle\bar{\psi} \psi\rangle$ and $f_{\pi}$ which are the chosen inputs of the model. The gap equation will be used in the determination of the coupling $G_{S}$ compatible with the mass fixed by the critical condition. At this point, it is crucial to ask: are the values for $M$ and $G_{S}$, emerging from this critical condition, reasonable?

In Fig. 1 we plot all possible physical solutions $(M>0)$ of Eq. (127) as a function of the arbitrary constant $C_{2}$. Using as input $\langle\bar{\psi} \psi\rangle=(-250.0 \mathrm{MeV})^{3}$ and $f_{\pi}=$ $93.0 \mathrm{MeV}$, it results that for values of $C_{2}<C_{\text {crit }}$, Eq. (127), there is no physical solution, at $C_{2}=C_{\text {crit }}$ there is only one solution, and for $C_{2}>C_{\text {crit }}$ there are two possible solutions. In particular, at the critical point we obtain $C_{\text {crit }} \simeq 24.82 \mathrm{MeV}^{2}, M \simeq 468.4 \mathrm{MeV}$, and $G_{S} \simeq$ $7.5 \mathrm{GeV}^{-2}$. Therefore the values for $M$ and $G_{S}$ are in good agreement with the ones found in the literature of this issue.

Another difference between this formulation and the traditional ones refers to the meson phenomenology. When we adopt the present formulation, in the presence of chiral symmetry breaking parameter $m_{0}$, previously fixed, since it is an input parameter, the meson masses and meson-quark-quark couplings, as well as other physical aspects, emerge as genuine predictions, including those of pion. In order to see this aspect clearly, let us consider the case with $m_{0} \neq 0$. As it can be seen in Eq. (112), the

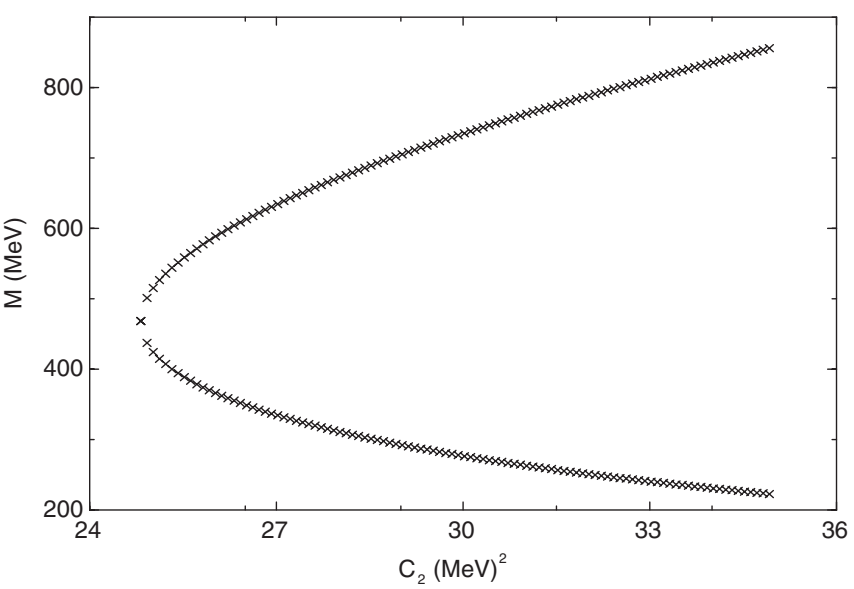

FIG. 1. Solutions of Eq. (127) as a function of the constant $C_{2}$.

pion mass is nonzero, reflecting the fact that the original Lagrangian is not chirally symmetric with $m_{0} \neq 0$. The introduction of a current quark mass modifies the results for other quantities as well. For example, the expression for $f_{\pi}$ now contains a finite part, and because of this, the expression of $I_{\log }\left(M^{2}\right)$ in terms of $f_{\pi}^{2}$ changes to

$$
\begin{aligned}
i\left[I_{\log }\left(M^{2}\right)\right]= & -\frac{\left[Z_{0}\left(m_{\pi}^{2}, M^{2}\right)\right]}{16 \pi^{2}}-\frac{f_{\pi}^{2}}{4 N_{c} N_{f} M^{2}} \\
& \times\left(1+\sqrt{1-\frac{N_{c} N_{f} m_{\pi}^{2} M^{2}\left[Y_{1}\left(m_{\pi}^{2}, M^{2}\right)\right]}{2 \pi^{2} f_{\pi}^{2}}}\right) .
\end{aligned}
$$

As a result, we can write for the pion mass the following expression:

$$
m_{\pi}^{2}=\frac{m_{0} M}{f_{\pi}^{2} G_{S}}\left(1+\sqrt{1-\frac{N_{c} N_{f} m_{\pi}^{2} M^{2}\left[Y_{1}\left(m_{\pi}^{2}, M^{2}\right)\right]}{2 \pi^{2} f_{\pi}^{2}}}\right)^{-1} .
$$

Also, for the effective pion-coupling constant we obtain

$$
\begin{aligned}
g_{\pi q q}^{-2}= & -\frac{N_{c} N_{f} m_{\pi}^{2}}{8 \pi^{2}}\left[Y_{1}\left(m_{\pi}^{2}, M^{2}\right)\right] \\
& +\frac{f_{\pi}^{2}}{2 M^{2}}\left(1+\sqrt{1-\frac{N_{c} N_{f} m_{\pi}^{2} M^{2}\left[Y_{1}\left(m_{\pi}^{2}, M^{2}\right)\right]}{2 \pi^{2} f_{\pi}^{2}}}\right) .
\end{aligned}
$$

As before, eliminating $I_{\text {quad }}\left(M^{2}\right)$ and $I_{\log }\left(M^{2}\right)$ in favor of $\langle\bar{\psi} \psi\rangle$ and $f_{\pi}$, we obtain a nonlinear equation for $M$ that now involves the pion mass $m_{\pi}$, 


$$
\begin{aligned}
\frac{M^{3}}{16 \pi^{2}}[1 & \left.+Z_{0}\left(m_{\pi}^{2}, M^{2}\right)\right] \\
= & \frac{\langle\bar{\psi} \psi\rangle}{4 N_{c}}+C_{2} M \\
& -\frac{f_{\pi}^{2} M}{4 N_{c} N_{f}}\left(1+\sqrt{1-\frac{N_{c} N_{f} m_{\pi}^{2} M^{2}\left[Y_{1}\left(m_{\pi}^{2}, M^{2}\right)\right]}{2 \pi^{2} f_{\pi}^{2}}}\right) .
\end{aligned}
$$

This equation is much more complicated to solve the one in the case of exact symmetry. This equation and the expression for $m_{\pi}$, however, can be simplified using the following approximations for $Z_{0}$ and $Y_{1}$ :

$$
Z_{0}\left(q^{2} ; m^{2}\right)=-\frac{q^{2}}{6 m^{2}}-\frac{q^{4}}{60 m^{4}}+\cdots
$$

and

$$
Y_{1}\left(q^{2} ; m^{2}\right)=-\frac{1}{6 m^{2}}-\frac{q^{2}}{30 m^{4}}+\cdots
$$

Using these approximations, we obtain

$$
\begin{gathered}
m_{\pi}^{2}=\frac{m_{0} M}{f_{\pi}^{2} G_{S}}\left(1+\sqrt{1+\frac{N_{c} N_{f} m_{\pi}^{2}}{12 \pi^{2} f_{\pi}^{2}}}\right)^{-1}, \\
\frac{M^{3}}{16 \pi^{2}}+\frac{f_{\pi}^{2} M}{4 N_{c} N_{f}}\left(1+\sqrt{1+\frac{N_{c} N_{f} m_{\pi}^{2}}{12 \pi^{2} f_{\pi}^{2}}}\right) \\
-\frac{m_{\pi}^{2} M}{96 \pi^{2}}-C_{2} M-\frac{\langle\bar{\psi} \psi\rangle}{4 N_{c}}=0 .
\end{gathered}
$$

Inserting the "experimental" value for $m_{0}$ (as well as for $\langle\bar{\psi} \psi\rangle$ and $f_{\pi}$ ) we search for the value of $C_{2}$ which corresponds to only one positive value for $M$ and thus determines the values for $G_{S}, m_{\pi}$, and so on. For this propose, we take $m_{0}=5.2 \mathrm{MeV}$, obtaining $m_{\pi} \simeq 135.3 \mathrm{MeV}, M \simeq$ $468.4 \mathrm{MeV}, G_{S} \simeq 7.5 \mathrm{GeV}^{-2}$, and $C_{\text {crit }} \simeq 24.82 \mathrm{MeV}^{2}$. Finally, Eq. (131) furnishes $g_{\pi q q} \simeq 4$.97. These predictions are in good agreement with experimental data and those used in the literature.

In the scalar channel, we have the following expressions for the $\sigma$ meson mass and the $\sigma q q$ coupling constant:

$$
\begin{aligned}
m_{\sigma}^{2}= & 4 M^{2}-\frac{m_{0}}{M G_{S}}\left[\frac{N_{c} N_{f}}{4 \pi^{2}}\left[Z_{0}\left(m_{\sigma}^{2}, M^{2}\right)+\frac{m_{\pi}^{2}}{6 M^{2}}\right]\right. \\
& \left.-\frac{f_{\pi}^{2}}{M^{2}}\left(1+\sqrt{1+\frac{N_{c} N_{f} m_{\pi}^{2}}{12 \pi^{2} f_{\pi}^{2}}}\right)\right]^{-1},
\end{aligned}
$$

$$
\begin{aligned}
g_{\sigma q q}^{-2}= & -\frac{N_{c} N_{f}}{8 \pi^{2}}\left[Z_{0}\left(m_{\sigma}^{2}, M^{2}\right)+\frac{m_{\pi}^{2}}{6 M^{2}}\right] \\
& -\frac{N_{c} N_{f}}{8 \pi^{2}}\left(m_{\sigma}^{2}-4 M^{2}\right)\left[Y_{1}\left(m_{\sigma}^{2}, M^{2}\right)\right] \\
& +\frac{f_{\pi}^{2}}{2 M^{2}}\left(1+\sqrt{1+\frac{N_{c} N_{f} m_{\pi}^{2}}{12 \pi^{2} f_{\pi}^{2}}}\right) .
\end{aligned}
$$

Numerically, we have $m_{\sigma}=938 \mathrm{MeV}$ and $g_{\sigma q q}=2.29$.

The fact of the arbitrary character of $C_{2}$ being removed, owing to the existence of a critical condition, it is the most important result point in the analysis made in this section. This means that the phenomenology becomes completely independent of the specific regularization scheme employed if such a scheme is consistent with the scale invariance.

The model, within the scope of this prescription, becomes predictive since the role played by a regularization has completely disappeared. In this sense, in spite of being a nonrenormalizable model, the predictions are made in the same spirit as in renormalized models, at the considered level of approximation. This is, undoubtedly, a very important improvement in the quality of this type of perturbative calculations. However, we must be aware of the fact that this does not represent the solution to all the problems involved. Since the amplitudes have acquired structures which are very similar to those belonging to the renormalized theories, what remains to be considered are the phenomenological implications for the model predictions of the so-called Landau vacuum instabilities or ghost poles in boson propagators. This aspect of the perturbative calculations appeared in hadron physics in connection with the Skyrme model [26-29]. More specifically, Landau vacuum instabilities were found in chiral-quark models when soliton solutions were searched for in the renormalized sigma model. (For an update of this problem, please see the recent paper of Arriola, Broniowski, and Golli [30]). However, Landau instabilities seem to be present in almost all renormalizable theories where fermions are coupled with boson fields [28]. Their occurrence can be indicated by the presence of tachyon poles in boson propagators corrected by one-loop fermionic contributions [31]. In asymptotically free theories where the bosons are selfinteracting fields, contributions coming from bosonic one-loop diagrams may eliminate the problem [31], otherwise the general rule seems to be the existence of vacuum instabilities [28]. In the QED, where the vector gauge field is not a self-interacting field, tachyonic poles occur in the photon propagator such that the vacuum is unstable at the one-loop level. In the linear sigma model, the meson propagators are equally contaminated by tachyonic poles. In the QED, the presence of such a type of undesirable poles does not play a physically relevant role because the scale of the fluctuations at which the instabilities occur is $m e^{1 / \sigma}$ ( $m$ is the electron mass and $\alpha$ is the fine structure constant) [28]. Therefore, this happens in a region which is 
certainly beyond the expected validity of the theory. On the other hand, in hadronic phenomenological models, where a fermionic field is coupled with a mesonic one, like in the Yukawa model or in the chiral $\sigma$ model, such scale of fluctuations changes drastically (around $1 \mathrm{GeV}$ ) due to the nucleon or quark mass and due to the value of the constant coupling involved. This means that the ghost poles may have relevant influence in phenomenological implications of the model.

In the NJL model, we have only fermions in the Lagrangian but the meson states are interpreted as quarkantiquark bound states. The intermediate amplitudes are fermionic loops such that, in the random-phase approximation, the meson mass is identified as the pole of Eq. (107). This is precisely the structure of the renormalized meson propagator in the linear $s$ model. This means that if we look carefully to the condition stating the pion mass, for example, we will find that in addition to the pion pole, there is a tachyonic pole in the Euclidian region with a negative residue. This implies that the corresponding dispersion relation will be verified only if this pole is included. This situation is not commonly considered within the context of the NJL model due to the use of cutoffs which change the behavior of the fermionic oneloop contributions such that the problem is very often automatically eradicated [30].

In the procedure adopted in the present work, the finite parts of the Green's functions are not modified, putting the physical amplitudes at the same level as those belonging to renormalizable theories. Because of this, the questions related to the ghost poles or vacuum instabilities may become relevant. The presence of ghost poles in meson propagators or Landau vacuum instabilities in the NJL model is expected due to its equivalence to the linear $\sigma$ model. Although the Green's functions are, strictly speaking, not the same ones, due to the definition of the renormalization parameters, the $S$ matrices of both theories are identical [2].

This aspect can be easily stated if we plot the denominator of the sigma or pion propagators looking for roots in the $q^{2}<0$ region. Let us consider the pion propagator at the random-phase approximation. In the Euclidean region $\left(q^{2}<0\right)$ the Eq. (111) may be written

$$
\begin{aligned}
\Pi_{\pi}\left(q^{2}\right)= & -\frac{2\langle\bar{\psi} \psi\rangle}{M}+\frac{f_{\pi}^{2}}{2 M^{2}} q^{2}\left(1+\sqrt{1+\frac{m_{\pi}^{2}}{2 \pi^{2} f_{\pi}^{2}}}\right) \\
& -\frac{3}{4 \pi^{2}} q^{2}\left\{\frac{m_{\pi}^{2}}{6 M^{2}}+\left[Z_{0}\left(q^{2}, M^{2}\right)\right]\right\},
\end{aligned}
$$

where

$$
Z_{0}\left(q^{2}, M^{2}\right)=-2+\sqrt{1+\frac{4 M^{2}}{q^{2}}} \ln \left(\frac{\sqrt{1+\frac{4 M^{2}}{q^{2}}}+1}{\sqrt{1+\frac{4 M^{2}}{q^{2}}}-1}\right) .
$$

In Fig. 2, we plot the denominator of the pion propagator,

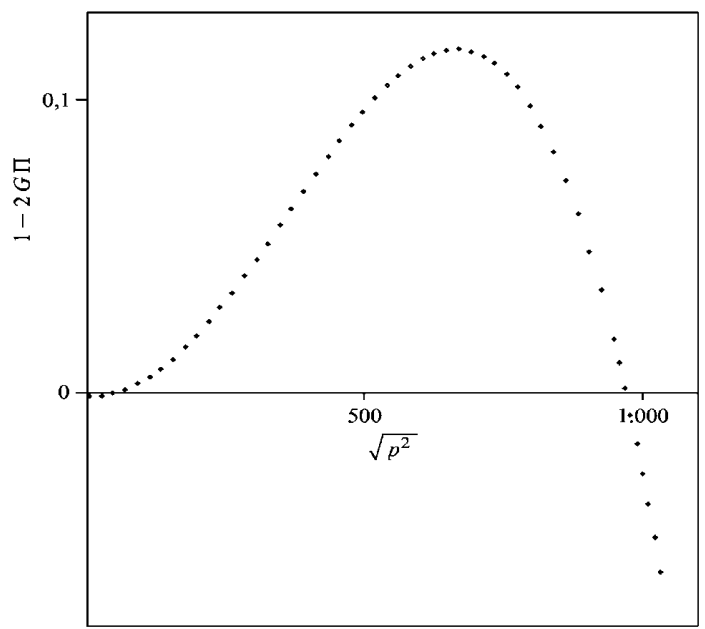

FIG. 2. Denominator of pion-propagator as a function of $q^{2}<$ 0 .

$1-2 G_{S} \Pi_{\pi}\left(q^{2}\right)$, in the Euclidean region. Now we can clearly see that there is a pole in the Euclidean region around $1 \mathrm{GeV}$. Similar results can be found for the sigma propagator.

On general grounds it is not completely clarified if ghost poles are real ingredients of quantum field theory (QFT) or if they are a product of a particular kind of perturbative solution (one-loop approximation) [28]. Because of the fact that if they are real ingredients of QFT, fundamental axioms are violated since only real poles are expected to exist. The relevant question seems to investigate if this type of instabilities survives to higher-order calculations. The acceptance that these undesirable poles are unavoidable and, therefore, real aspects of QFT, constitutes a very frustrating fact just because we are accepting that the solutions we can obtain do not obey the fundamental axioms of the theoretical apparatus we have constructed. The most reasonable expectation is that the instabilities will disappear when contributions of higher order are computed. If this is the case, there is nothing else to do but to conclude that the one-loop approximations are not adequate to investigate phenomenological implications of a theory or model. If, however, the instabilities constitute an unavoidable aspect of certain classes of QFT, we must make efforts to get theories free from those problems by construction, as in the case of anomalies. Only additional investigations will clarify these doubts. We are, however, convinced that the questions related to the regularizations in QFT are of a different nature from those related to the ghost poles in perturbative corrections of boson propagators.

\section{CONCLUSIONS AND PERSPECTIVES}

We considered in detail questions relative to the predictive power of the NJL model. Given its nonrenormalizability, the model predictions are usually compromising 
with the regularization method employed. The regularization cannot be removed from the results and consequently, the physical implications are crucially dependent on the adopted regularization technique. It is usual practice to consider the regularization as part of the model, see for example $[5,6,8]$. However, depending on the adopted regularization method, physical amplitudes may emerge from the calculations ambiguous and symmetry violating. Undefined quantities arising from divergences are fixed in the parametrization of the model by adjustments in regularization parameters. In view of this, one has the potential problem that the results of calculations are not real predictions, but particular choices for the involved arbitrariness (ambiguities) [16,17] that lead to results that might change when different choices are made.

Our investigation in the present paper focused on avoiding as much as possible explicit evaluations of divergent quantities. Our discussion consisted in basically two steps. In the first step, we obtained physical amplitudes free from ambiguities and symmetry preserving. Integrands of divergent Feynman integrals were manipulated such that all the dependence on internal (arbitrary) momenta is left in terms that lead to finite integrals, which are then integrated free from the regularization effects, while purely divergent objects (combinations of divergent integrals) can be clearly identified. Invoking symmetry constraints and demanding elimination of ambiguities lead to what we called consistency relations, which require definite values for the divergent objects. All one- and two-point functions of the model, in which the highest degrees of divergence reside, emerge from the calculations free from ambiguities and respecting symmetry constraints. In the second step, a parametrization was introduced to eliminate divergences. We considered a parametrization of the model where the remaining divergent integrals, $I_{\text {quad }}\left(M^{2}\right)$ and $I_{\log }\left(M^{2}\right)$, need to be eliminated by fixing phenomenological quantities. We used general scale properties of $I_{\text {quad }}\left(M^{2}\right)$ and $I_{\log }\left(M^{2}\right)$ in order to make explicit the freedom one always has when choosing a specific regularization through the constant parameter $C_{2}$. All this was done without explicit evaluation of the divergent integrals.

Of course, we could also have chosen a specific regularization for evaluating explicitly $I_{\text {quad }}\left(M^{2}\right)$ and $I_{\log }\left(M^{2}\right)$ and put our calculation in close connection with the traditional regularization methods. As a result, both $I_{\text {quad }}\left(M^{2}\right)$ and $I_{\log }\left(M^{2}\right)$ become a function of a regularization parameter $\Lambda$ and would also depend on the form chosen for the regularization function $G\left(k^{2} / \Lambda^{2}\right)$. In this way, for different regularizations one would have different values for $C_{2}$. We have shown that without making any choice, one can fix this $C_{2}$ by simply choosing its critical value. An important point to be noted, we reiterate, is that all manipulations done prior to such a choice have been made guaranteed amplitudes that are free from loop momenta ambiguities and that preserve the symmetries of the model.

Just due to this fact, we have denominated the formulation presented here as predictive. The assertion that the formulation of the regularization of the NJL model presented here is predictive is to be understood in the sense that no arbitrary choices were made in intermediate steps up the stage of calculating phenomenological quantities, like the pion and sigma masses and coupling constants. Even at this last stage, the existence of a critical value for $\mathrm{C}_{2}$ that leads to good values for phenomenological quantities is gratifying. One more time, however, we point out that the procedure adopted in the present work does not solve all the problems involved, as well as does not guarantee that the predictions are in excellent agreement with all the experimental data pertinent to the model scope. All we can say is that the predictions are not dependent on arbitrary choices involved in the calculation of amplitudes. Intrinsic problems of the perturbative calculations as the ghost poles or the associated vacuum instabilities must be studied, and their implications for the phenomenology clearly stated. Perhaps the association of the procedure adopted in the present work to treat the amplitudes with procedures devoted to the elimination of vacuum instabilities may give us adequate answer to such questions [32]. Having this in mind, it would be interesting to apply the formulation of the NJL model presented here to the SU(3) version. Work along this line is presently under way.

\section{ACKNOWLEDGMENTS}

This work was partially supported by the Brazilian agencies CNPq and FAPESP.
[1] Y. Nambu and G. Jona-Lasinio, Phys. Rev. 122, 345 (1961); 124, 246 (1961).

[2] T. Eguchi, Phys. Rev. D 14, 2755 (1976).

[3] K. Kikkawa, Prog. Theor. Phys. 56, 947 (1976).

[4] U. Vogl and W. Weise, Prog. Part. Nucl. Phys. 27, 195 (1991).

[5] S. P. Klevansky, Rev. Mod. Phys. 64, 649 (1992).

[6] T. Hatsuda and T. Kunihiro, Phys. Rep. 247, 221 (1994).
[7] Chr. V. Christov, A. Blotz, H.-C. Kim, P. Pobylitsa, T. Watabe, T. Meissner, E. Ruiz Arriola, and K. Goeke, Prog. Part. Nucl. Phys. 37, 91 (1996).

[8] J. Bijnens, Phys. Rep. 265, 369 (1996).

[9] V. Bernard, A. H. Blin, B. Hiller, Y.P. Ivanov, A. A. Osipov, and U.-G. Meissner, Ann. Phys. (N.Y.) 249, 499 (1996).

[10] G. Ripka, in Quarks Bound by Chiral Fields (Oxford 
Science Publications, London, 1997).

[11] M. Buballa, Phys. Rep. 407, 205 (2005).

[12] S. Weinberg, Physica A (Amsterdam) 96, 327 (1979).

[13] W. Broniowski, G. Ripka, E. Nikolov, and K. Goeke, Z. Phys. A 354, 421 (1996).

[14] F. Doring, A. Blotz, C. Schuren, T. Meissner, E. Ruiz Arriola, and K. Goeke, Nucl. Phys. A536, 548 (1992).

[15] R. M. Davidson and E. Ruiz Arriola, Acta Phys. Pol. B 33, 1791 (2002); Phys. Lett. B 359, 273 (1995); 348, 163 (1995).

[16] R. S. Willey, Phys. Rev. D 48, 2877 (1993).

[17] T. Gherghetta, Phys. Rev. D 50, 5985 (1994).

[18] O. A. Battistel and M. C. Nemes, Phys. Rev. D 59, 055010 (1999).

[19] O.A. Battistel, Ph.D. thesis, Universidade Federal de Minas Gerais, Brazil, 1999.

[20] O. A. Battistel and G. Dallabona, Nucl. Phys. B610, 316 (2001).

[21] O. A. Battistel and O. L. Battistel, Int. J. Mod. Phys. A 17,
1979 (2002).

[22] G. Dallabona and O. A. Battistel, Phys. Rev. D 70, 065017 (2004).

[23] O. A. Battistel and G. Dallabona, J. Phys. G 28, 2539 (2002).

[24] O. A. Battistel and G. Dallabona (work in progress).

[25] O. A. Battistel (unpublished).

[26] G. Ripka and S. Kahana, Phys. Rev. D 36, 1233 (1987).

[27] V. Soni, Phys. Lett. B 183, 91 (1987).

[28] T. D. Cohen, M. K. Banerjee, and Ching-Yun Ren, Phys. Rev. C 36, 1653 (1987).

[29] T. Meissner, E. Ruiz Arriola, F. Grummer, H. Mavromatis, and K. Goeke, Phys. Lett. B 214, 312 (1988).

[30] E. Ruiz Arriola, W. Broniowski, and B. Golli, Phys. Rev. D 76, 014008 (2007).

[31] R. J. Perry, Phys. Lett. B 199, 489 (1987).

[32] J. Caro, E. Ruiz Arriola, and L. L. Salcedo, Phys. Rev. C 55, 1767 (1997). 